\title{
WELL-POSEDNESS AND ASYMPTOTIC BEHAVIOR OF A NONAUTONOMOUS, SEMILINEAR HYPERBOLIC- PARABOLIC EQUATION WITH DYNAMICAL BOUNDARY CONDITION OF MEMORY TYPE
}

\author{
HASSAN YASSINE
}

\author{
Communicated by Stig-Olof Londen
}

\begin{abstract}
We consider a nonautonomous, semilinear, hyperbolic-parabolic equation subject to a dynamical boundary condition of memory type. First we prove the existence and uniqueness of global bounded solutions having relatively compact range in the natural energy space. Under the assumption that the nonlinear term $f$ is real analytic, we then derive an appropriate Lyapunov energy and we use the Łojasiewicz-Simon inequality to show the convergence of global weak solutions to single steady states as time tends to infinity. Finally, we provide an estimate for the convergence rate.
\end{abstract}

1. Introduction. The main purpose of this work is to study the existence and the asymptotic behavior of global weak solutions to the semilinear degenerate wave equation with boundary conditions of memory type given by

$$
\begin{cases}K_{1}(x) u_{t t}+K_{2}(x) u_{t}-\Delta u+f(x, u)=g_{1} & \text { in } \mathbf{R}^{+} \times \Omega, \\ \partial_{\nu} u+\mu(x) u+k * u_{t}=g_{2} & \text { on } \mathbf{R}^{+} \times \Gamma, \\ u(0)=u_{0}, \sqrt{K_{1}} u_{t}(0)=\sqrt{K_{1}} u_{1} . & \end{cases}
$$

Here, $\Omega \subseteq \mathbf{R}^{N}(N \geq 1)$ is a bounded open connected set with smooth boundary $\Gamma, \nu$ denotes the outer normal vector to the boundary. The coefficients $K_{1}, K_{2} \in L^{\infty}(\Omega), \mu \in W^{1, \infty}(\Gamma)$ and $k \in L_{\text {loc }}^{1}\left(\mathbf{R}^{+}\right)$are nonnegative functions, $K_{2}(x) \geq k_{0}>0, \mu$ is not identically zero on $\Gamma$, and $k * v$ stands for the convolution on the positive half-line, that is, $(k * v)(t)=\int_{0}^{t} k(t-s) v(s) d s(t \geq 0)$.

2010 AMS Mathematics subject classification. Primary 28C15, 28C99, 46E05.

Keywords and phrases. Evolutionary integral equation, semilinear, dynamic boundary condition, stabilization, Łojasiewicz-Simon inequality.

Received by the editors on August 18, 2012, and in revised form on March 24, 2013.

DOI:10.1216/JIE-2013-25-4-517 Copyright (C)2013 Rocky Mountain Mathematics Consortium 
The boundary condition arises in mathematical models for the motion of viscoelastic materials. For such materials, the feedback operator is a convolution operator in time. We consider also the case in which the kernel is singular; a typical example for the kernel $k$ we have in mind is given by

$$
k(t)=\frac{1}{\Gamma(1-\beta)} t^{-\beta} e^{-w t} \quad(\beta \in(0,1), w>0),
$$

where $\Gamma$ is the Gamma function.

The nonlinearity $f=f(x, u): \Omega \times \mathbf{R} \rightarrow \mathbf{R}$ is assumed to be a $C^{2}$ function satisfying the following assumptions:

$(F 1)$ The function $f$ is analytic in the second variable, uniformly with respect to $x \in \Omega$ and $u$ in bounded subsets of $\mathbf{R}$;

$(F 2)$ One has $f(\cdot, 0) \in L^{\infty}(\Omega)$, and there exist constants $\rho \geq 0$ and $\alpha \in(0,1],(N-2) \alpha<2$ such that:

$$
\left|\frac{\partial f}{\partial u}(x, u)\right| \leq \rho\left(1+|u|^{\alpha}\right) \quad \text { for every } u \in \mathbf{R}, \quad x \in \Omega .
$$

(F3) There exist $\lambda<\lambda_{1}$ and $C \geq 0$ such that, for every $u \in \mathbf{R}$ and every $x \in \Omega$,

$$
F(x, u) \geq-\lambda \frac{u^{2}}{2}-C,
$$

where $F(x, u)=\int_{0}^{u} f(x, s) d s(x \in \Omega, u \in \mathbf{R})$, and $\lambda_{1}>0$ is the best Sobolev constant in the following Poincaré type inequality

$$
\int_{\Omega}|\nabla u|^{2}+\int_{\Gamma} \mu(x)|u|^{2} \geq \lambda_{1} \int_{\Omega}|u|^{2} \quad\left(u \in H^{1}(\Omega)\right) .
$$

We study well-posedness of equation (1) in the energy space $\mathcal{H}=$ $H^{1}(\Omega) \times L^{2}(\Omega)$ and the asymptotic behavior of weak solutions when $t \rightarrow \infty$. In particular, for all initial values in the natural energy space, we prove the existence and uniqueness of a global, bounded solution of (1). In addition, we prove that every global, bounded solution has relatively compact range in $\mathcal{H}$. Then, by using a new Lyapunov functional and the Łojasiewicz-Simon inequality, we show that, if $g_{1}$ and $g_{2}$ tend to 0 sufficiently fast at infinity, then the solution of (1) 
converges to a single steady state. Finally, we show that the decay rate to equilibrium is either exponential or polynomial.

Concerning existence of solutions, we carefully note that the function $K_{1}$ may vanish on $\Omega$ or on a subset of $\Omega$. Equation (1) thus includes the semilinear diffusion equation $\left(K_{1}=0\right)$, the semilinear wave equation $\left(K_{1}=1\right)$, and mixed hyperbolic-parabolic problems $\left(K_{1} \geq 0\right)$. In our existence proof below, we shall first replace $K_{1}$ by $K_{1}+\varepsilon$ and prove existence of solutions for this perturbed, purely hyperbolic problem by means of a Faedo-Galerkin method. We shall further obtain a priori estimates for the solutions which are independent of $\varepsilon>0$, in such a way that we can pass to the limit when $\varepsilon$ tends to zero, obtaining thus a function $u$ which is the solution of problem (1). By differentiating the equation with respect to time, we shall also prove the existence of strong solutions if the data are regular enough.

We recall that the basic argument in the proof of the convergence results is the Eojasiewicz inequality which was generalized first by Simon [19], then by Haraux and Jendoubi $[\mathbf{1 4}, \mathbf{1 6}, \mathbf{1 7}$ ] (see below for the definition of the Łojasiewicz-Simon inequality).

Concerning the convergence to steady state for nonlinear equations with memory there is a technical difficulty consisting in proving that the solutions of such problems are bounded and have relatively compact range in the natural energy space. However, the more complicated problem is to find an appropriate Lyapunov functional in order to investigate the asymptotic behaviour of global, bounded solutions. For the type of kernel $k$ and nonlinearity $f$ as above, we note that there are up to now two techniques to construct an appropriate Lyapunov functional which allows one to apply the Łojasiewicz-Simon inequality in order to obtain a convergence result. The first technique goes back to Dafermos [10], and this technique was recently adapted by Aizicovici and Feireisl [1] in order to obtain a convergence result for a phase-field model with memory (see also Aizicovici and Petzeltová [2]), and then by Chill and Fašangová [8] in order to obtain convergence results for the wave equation, where the dissipation is both frictional and with memory:

$$
u_{t t}+u_{t}+k * u_{t}-\Delta u+f(x, u)=0 \quad \text { in } \mathbf{R}^{+} \times \Omega .
$$

Recently, Zacher and Vergara [20] have developed a second technique to find Lyapunov functions for ordinary differential equations, in finite- 
dimensional spaces, of order less than 1, and of order between 1 and 2 in time, which, combined with the Łojasiewicz inequality, leads to a proof of convergence of global, bounded solutions to a single steady state.

In [23], Zacher has proved that, still in the finite-dimensional case, the dissipation given through the memory term is strong enough to guarantee convergence of global, bounded and regular solutions of the following second order equation

$$
\ddot{u}+k * \dot{u}+\nabla E(u)=g,
$$

when the nonlinear potential $E$ satisfies the Eojasiewicz inequality. In his proof, Zacher used the Lojasiewicz inequality together with the method of higher order energies. In this direction it is important to mention the work of Alabau-Boussouira, Prüss and Zacher [3], also, where the autonomous, linear case $\left(f=K_{2}=g_{1}=g_{2}=0, K_{1}=1\right)$ was studied under the same boundary condition.

Concerning the nonautonomous, nonlinear case, the source terms introduce non-standard difficulties. The convergence proof given here is direct and naturally generalizes the autonomous case, without using the additional discussion from Chill and Jendoubi [9] or the additional integral lemma from Huang and Takác [15] (see also Feireisl and Simondon [11] and the author's article [21]).

Remark 1 (Related boundary conditions). For the well-posedness of the Robin-type problem, we assume that the coefficient $\mu$ on the boundary is not identically zero almost everywhere on $\Gamma$ (with respect to the surface measure). However, the following variants of (dynamical) boundary conditions may also be studied. Assume, for example, that $\Gamma=\Gamma_{0} \cup \Gamma_{1}$ for two closed, disjoint subsets $\Gamma_{0}, \Gamma_{1} \subseteq \Gamma$. Then the results of this paper (existence and uniqueness of global, bounded solutions, relative compactness of their range in the energy space, convergence to equilibrium and decay rate estimates) still hold for the following boundary condition

$$
\begin{cases}u=0 & \text { on } \mathbf{R}^{+} \times \Gamma_{0}, \\ \partial_{\nu} u+\mu(x) u+k * u_{t}=g_{2} & \text { on } \mathbf{R}^{+} \times \Gamma_{1}\end{cases}
$$


where $\mu \in W^{1, \infty}\left(\Gamma_{1}\right)$ is such that

$$
\left\{\begin{array}{l}
\text { if } \Gamma_{0} \neq \varnothing, \text { then } \mu \geq 0, \\
\text { if } \Gamma_{0}=\varnothing, \text { then } \mu \text { is not identically zero } \\
\text { almost everywhere on } \Gamma_{1} .
\end{array}\right.
$$

Also, the results of this paper still hold when the boundary feedback is both frictional and of memory type

$$
\begin{cases}u=0 & \text { on } \mathbf{R}^{+} \times \Gamma_{0}, \\ \partial_{\nu} u+\mu(x) u+b(x) u_{t}+k * u_{t}=g_{2} & \text { on } \mathbf{R}^{+} \times \Gamma_{1},\end{cases}
$$

where $b$ is a nonnegative function on $\Gamma_{1}$ and $\mu \in W^{1, \infty}\left(\Gamma_{1}\right)$ satisfying (4). This boundary condition has been studied in [3], when $g_{2}=0$; see also $[\mathbf{2 1}, \mathbf{2 2}$ ], when the feedback is only frictional (that is, $k=0$ ).

Another boundary condition, with more regular kernels, has been studied by several authors (see, for example, Santos [18], Cavalcanti et al. $[6,7]$ and the references therein), namely, the boundary condition

$$
\begin{cases}u=0 & \text { on } \mathbf{R}^{+} \times \Gamma_{0} \\ u+h * \partial_{\nu} u=0 & \text { on } \mathbf{R}^{+} \times \Gamma_{1}\end{cases}
$$

Here, the relaxation function $h$ belongs to $W^{1, \infty}(0, \infty)$ and is assumed to be positive and non-increasing. By differentiating equation (6) and by applying the inverse Volterra operator, we obtain

$$
\partial_{\nu} u=-\rho\left(u_{t}+k_{1}(0) u-k_{1}(t) u_{0}+k_{1}^{\prime} * u\right) \quad \text { on } \mathbf{R}^{+} \times \Gamma_{1},
$$

where $\rho=1 / h(0)$, and $k_{1}$ is the resolvent kernel satisfying

$$
k_{1}+\rho h^{\prime} * k_{1}=-\rho h^{\prime} .
$$

Observe that

$$
k_{1}^{\prime} * u=\frac{d}{d t}\left(k_{1} * u\right)-k_{1}(0) u=k_{1} * u_{t}+k_{1}(t) u_{0}-k_{1}(0) u .
$$

Then, the boundary condition (6) can be rewritten in the following form

$$
\begin{cases}u=0 & \text { on } \mathbf{R}^{+} \times \Gamma_{0} \\ \partial_{\nu} u+\rho u_{t}+\rho k_{1} * u_{t}=0 & \text { on } \mathbf{R}^{+} \times \Gamma_{1}\end{cases}
$$


which is a particular case of the boundary condition (5).

Throughout the following:

- The inner product (respectively the norm) in the spaces $H^{1}(\Omega)$, $H^{1}(\Omega)^{\prime}, L^{2}(\Omega)$ and $L^{2}(\Gamma)$ is denoted by $(\cdot, \cdot)_{H^{1}(\Omega)},(\cdot, \cdot)_{*},(\cdot, \cdot)_{2}$ and $(\cdot, \cdot)_{\Gamma}$ (respectively, by $\|\cdot\|_{H^{1}(\Omega)},\|\cdot\|_{*},\|\cdot\|_{2}$, and $\left.\|\cdot\|_{\Gamma}\right)$. The norm in $L^{p}(\Omega)$ is denoted by $\|\cdot\|_{p}$.

- We denote by $C$ (sometimes $C_{i}$ ) a generic positive constant which may vary from line to line, which may depend on $g_{1}, g_{2}, f$ and the measure of $\Omega$, but which can be chosen independently of $t \in \mathbf{R}^{+}$.

The remaining part of this paper is organized as follows. In Section 2, we state the assumptions on the kernel and the source terms, and we state the main results. The existence and uniqueness of solutions to problem (1) is proved in Section 3. Section 4 is devoted to the compactness results. In the final Section 5 , we prove the convergence of global bounded solutions and we obtain an estimate on the convergence rate.

2. Assumptions and main results. Before stating our main results, we present several assumptions about the initial data, the source terms, and the memory kernel.

2.1. Assumptions on the source terms and the kernel. For the global existence and uniqueness for weak solutions, we assume that the functions $g_{1}$ and $g_{2}$ satisfy the regularity condition

$$
g_{1} \in L_{\mathrm{loc}}^{2}\left(\mathbf{R}^{+} ; L^{2}(\Omega)\right) \text { and } g_{2} \in W^{1,2}\left(\mathbf{R}^{+} ; L^{2}(\Gamma)\right),
$$

and for our convergence result we assume in addition a decay condition, namely, that there exist constants $\eta_{0} \geq 0$ and $\delta>0$ such that for all $t \in \mathbf{R}^{+}$

$$
\left\|g_{2}(t)\right\|_{\Gamma}+\int_{t}^{\infty}\left(\left\|g_{1}(s)\right\|_{2}^{2}+\left\|g_{2}^{\prime}(s)\right\|_{\Gamma}^{2}\right) d s \leq \frac{\eta_{0}}{(1+t)^{1+\delta}} .
$$

Condition (G2) implies in turn that $g_{2} \in L^{1}\left(\mathbf{R}^{+} ; L^{2}(\Gamma)\right),\left\|g_{2}(t)\right\|_{\Gamma} \searrow 0$ and there exists an $\eta \geq 0$ such that

$$
\int_{t}^{\infty}\left(\left\|g_{1}(s)\right\|_{2}^{2}+\left\|g_{2}(s)\right\|_{\Gamma}^{2}+\left\|g_{2}^{\prime}(s)\right\|_{\Gamma}^{2}\right) d s \leq \frac{\eta}{(1+t)^{1+\delta}}
$$


Concerning the kernel $k$ we suppose that

(K1) there exists a nonnegative and nonincreasing kernel $b \in L_{\text {loc }}^{1}\left(\mathbf{R}^{+}\right)$ such that $b * k=1$, and

(K2) there are $\gamma>0$ and $a \in L^{1}\left(\mathbf{R}^{+}\right)$, strictly positive and nonincreasing, such that $b=a+\gamma(1 * a)$.

Remark 2. (a) Condition (K1) implies that the kernel $k$ is nonnegative.

(b) The conditions (K1) and (K2) together imply that $b(t) \geq b_{\infty}=$ $\lim _{s \rightarrow \infty} b(s)=\gamma\|a\|_{L^{1}\left(\mathbf{R}^{+}\right)}>0$ for every $t>0$.

(c) It follows further from conditions (K1) and (K2) that $k \in L^{1}\left(\mathbf{R}^{+}\right)$. Indeed, since $k$ is nonnegative (see (a)), the condition (K1) implies $(b * k)(t) \leq 1$ for every $t \geq 0$. Using the lower bound for $b$ from (b) and the positivity of $k$, we see that $\|k\|_{L^{1}\left(\mathbf{R}^{+}\right)} \leq 1 / b_{\infty}$.

(d) For each $\gamma>0$ the unique solution of the equation in (K2) is given by

$$
a=b-\gamma\left(e^{-\gamma \cdot} * b\right) .
$$

(e) Typical examples for the kernels $b$ and $k$ which satisfy conditions (K1) and (K2) are given by

$$
b(t)=\Gamma_{1-s}(t) e^{-w t}+w\left[1 *\left(\Gamma_{1-s} e^{-w}\right)(t)\right] \quad(s \in(0,1), w>0),
$$

$k$ and $\Gamma$ are given by $(2)$.

In fact, our method can be adapted to the more general case when the kernel $k$ is completely positive, that is, the condition (K1) can be weakened to the condition that

$\left(\mathrm{K} 1^{\prime}\right)$ there exist $b_{0}>0$ and a nonnegative and nonincreasing kernel $b \in L_{\text {loc }}^{1}\left(\mathbf{R}^{+}\right)$such that $b_{0} k(t)+(b * k)(t)=1$ for all $t \geq 0$ (see [23]).

This condition allows one to include the nonsingular case $s=0$ in example (2). In particular, our results are still valid for $k(t)=e^{-w t}$ $(t \geq 0, w>0)$.

2.2. Existence and uniqueness of global, bounded solutions. Throughout the following, a function $u: \mathbf{R}^{+} \rightarrow H^{2}(\Omega)$ is called a global 
strong solution of (1), if

$$
\left\{\begin{array}{l}
u \in L_{\mathrm{loc}}^{\infty}\left(\mathbf{R}^{+} ; H^{2}(\Omega)\right) \cap W_{\mathrm{loc}}^{1, \infty}\left(\mathbf{R}^{+} ; H^{1}(\Omega)\right) \cap W_{\mathrm{loc}}^{2,2}\left(\mathbf{R}^{+} ; L^{2}(\Omega)\right) \\
K_{1}^{1 / 2} u_{t} \in W_{\mathrm{loc}}^{1, \infty}\left(\mathbf{R}^{+} ; L^{2}(\Omega)\right)
\end{array}\right.
$$

if it satisfies the initial conditions $u(0)=u_{0}$ and $\left(K_{1}\right)^{1 / 2} u_{t}(0)=$ $\left(K_{1}\right)^{1 / 2} u_{1}$, and if it satisfies the differential equation (1) almost everywhere on $\mathbf{R}^{+}$. A function $u \in C\left(\mathbf{R}^{+} ; H^{1}(\Omega)\right) \cap W_{\mathrm{loc}}^{1,2}\left(\mathbf{R}^{+} ; L^{2}(\Omega)\right)$ is called a global weak solution of $(1)$, if it satisfies the initial conditions $u(0)=u_{0}$ and $\left(K_{1}\right)^{1 / 2} u_{t}(0)=\left(K_{1}\right)^{1 / 2} u_{1}$, and if there exists a sequence $\left(u^{\mu}\right)$ of strong solutions such that

$$
\begin{aligned}
u^{\mu} \longrightarrow u & \text { in } C\left(\mathbf{R}^{+} ; H^{1}(\Omega)\right) \cap W_{\mathrm{loc}}^{1,2}\left(\mathbf{R}^{+} ; L^{2}(\Omega)\right), \\
K_{1}^{1 / 2} u_{t}^{\mu} \longrightarrow K_{1}^{1 / 2} u_{t} & \text { in } C\left(\mathbf{R}^{+} ; L^{2}(\Omega)\right) .
\end{aligned}
$$

Our first main result, which establishes the global well-posedness of equation (1), reads as follows.

Theorem 3(Existence and uniqueness of global, bounded solutions). Assume that the function $f$ satisfies conditions $(\mathrm{F} 2)$ and $(\mathrm{F} 3)$, and that the kernel $k$ satisfies conditions (K1) and (K2).

(I) Strong solutions. Let

$g_{1} \in W_{\mathrm{loc}}^{1,2}\left(\mathbf{R}^{+} ; L^{2}(\Omega)\right) \quad$ and $\quad g_{2} \in L_{\mathrm{loc}}^{1}\left(\mathbf{R}^{+} ; H^{\frac{1}{2}}(\Gamma)\right) \cap W^{2,2}\left(\mathbf{R}^{+} ; L^{2}(\Gamma)\right)$,

and let the initial values $\left(u_{0}, u_{1}\right) \in H^{2}(\Omega) \times H^{q}(\Omega),(q>3 / 2)$, satisfy the compatibility conditions

$$
\begin{cases}-\Delta u_{0}+f\left(x, u_{0}\right)=g_{1}(0)-K_{2} u_{1} & \text { in } \Omega, \\ \partial_{\nu} u_{0}+\mu(x) u_{0}=g_{2}(0) & \text { on } \Gamma .\end{cases}
$$

Then problem (1) possesses a unique, global, strong solution.

(II) Weak solutions. Let $g_{1}$ and $g_{2}$ satisfy the regularity condition (G1) and let $\left(u_{0}, u_{1}\right) \in \bar{D}$, where

$$
D=\left\{\left(u_{0}, u_{1}\right) \in H^{2}(\Omega) \times H^{q}(\Omega) ; q>\frac{3}{2} \text { and (9) holds }\right\} .
$$


Then problem (1) possesses a unique global weak solution $u$. In addition, this weak solution satisfies the following properties:

(T1) $\left(u, K_{1}^{1 / 2} u_{t}\right)$ is bounded in $H^{1}(\Omega) \times L^{2}(\Omega)$.

(T2) $\left(u_{t}, v\right) \in L^{2}\left(\mathbf{R}^{+} ; L^{2}(\Omega)\right) \times L^{2}\left(\mathbf{R}^{+} ; L^{2}(\Gamma)\right)$, where $v=(d / d t)(k *$ $\left.\left(u-u_{0}\right)\right)$.

(T3) Let $G: \mathbf{R}^{+} \rightarrow \mathbf{R}$ be the energy of the solution $u$ given by

$$
\begin{aligned}
G(t)= & \frac{1}{2}\left\|K_{1}^{1 / 2} u_{t}\right\|_{2}^{2}+E(u)+\frac{1}{2} a *\|v\|_{\Gamma}^{2}-\left(g_{2}, a * v\right)_{\Gamma} \\
& +\frac{1}{2 k_{0}} \int_{t}^{\infty}\left\|g_{1}(s)\right\|_{2}^{2} d s \\
& +d \int_{t}^{\infty}\left(\left\|g_{2}(s)\right\|_{\Gamma}^{2}+\left\|g_{2}^{\prime}(s)\right\|_{\Gamma}^{2}\right) d s,
\end{aligned}
$$

where $d=\|a\|_{L^{1}\left(\mathbf{R}^{+}\right)} \max \left(\gamma, \gamma^{-1}\right)$ and $E: H^{1}(\Omega) \rightarrow \mathbf{R}$ is the energy functional given by

$$
E(u)=\frac{1}{2} \int_{\Omega}|\nabla u|^{2} d x+\int_{\Omega} F(x, u) d x+\frac{1}{2} \int_{\Gamma} \mu(x)|u|^{2} d \sigma .
$$

Then $G$ is nonincreasing and

$$
\frac{d}{d t} G(t) \leq-\frac{k_{0}}{2}\left\|u_{t}\right\|_{2}^{2}-\frac{b_{\infty}}{2}\|v\|_{\Gamma}^{2}-\frac{\gamma}{4} a *\|v\|_{\Gamma}^{2}, \quad t>0 .
$$

(T4) The following variational equality holds for all $\phi \in H^{1}(\Omega)$

$$
\begin{aligned}
\frac{d}{d t} \int_{\Omega} K_{1}(x) u_{t} \phi d x+\int_{\Omega} & K_{2}(x) u_{t} \phi d x \\
& +\int_{\Omega} \nabla u \nabla \phi d x+\int_{\Omega} f(x, u) \phi d x \\
& +\frac{d}{d t} \int_{\Gamma}\left(k *\left(u-u_{0}\right)\right) \phi d \sigma+\int_{\Gamma} \mu(x) u \phi d \sigma \\
= & \int_{\Omega} g_{1} \phi d x+\int_{\Gamma} g_{2} \phi d \sigma .
\end{aligned}
$$

Remark 4. (a) When $K_{1}=$ const, we replace the compatibility conditions (9) by the compatibility conditions

$$
\partial_{\nu} u_{0}+\mu(x) u_{0}=g_{2}(0) \quad \text { on } \quad \Gamma \text {. }
$$


(b) Note that, for every $u_{1} \in L^{2}(\Omega)$, the problem (9) admits at most one solution $u_{0} \in H^{2}(\Omega)$.

2.3. Compactness property of solutions. In the following theorem, we state an additional property of global weak solutions of (1) which is of crucial importance for the study of their asymptotic behavior, namely, the relative compactness of their range.

Theorem 5. Let $u: \mathbf{R}^{+} \rightarrow H^{1}(\Omega)$ be a global bounded weak solution of (1). Then the function $U=\left(u, K_{1}^{1 / 2} u_{t}\right)$ is uniformly continuous from $\mathbf{R}^{+}$with values in $H^{1}(\Omega) \times L^{2}(\Omega)$, and $\bigcup_{t \geq 0}\{U(t)\}$ is relatively compact in $H^{1}(\Omega) \times L^{2}(\Omega)$.

\subsection{The Łojasiewicz-Simon inequality for the underlying} energy. Our basic argument in the proof of the convergence result below is the Lojasiewicz-Simon inequality for the energy functional $E$ given by (11). By the regularity and growth condition on $f$, the function $E$ is twice continuously Fréchet differentiable $[\mathbf{2 1}]$. Let $E^{\prime}(u) \in H^{1}(\Omega)^{\prime}$ and $E^{\prime \prime}(u) \in \mathcal{L}\left(H^{1}(\Omega), H^{1}(\Omega)^{\prime}\right)$ denote the first and second derivative at a point $u \in H^{1}(\Omega)$, respectively. Then, for all $\phi$, $\psi \in H^{1}(\Omega)$

$$
\begin{aligned}
\left(E^{\prime}(u), \psi\right)_{H^{1}(\Omega)^{\prime}, H^{1}(\Omega)}= & \int_{\Omega} \nabla u \nabla \psi d x+\int_{\Omega} f(x, u) \psi d x \\
& +\int_{\Gamma} \mu(x) u \psi d \sigma
\end{aligned}
$$

and

$$
\begin{aligned}
\left(E^{\prime \prime}(u) \phi, \psi\right)_{H^{1}(\Omega)^{\prime}, H^{1}(\Omega)}= & \int_{\Omega} \nabla \phi \nabla \psi d x+\int_{\Omega} \frac{\partial f}{\partial u}(x, u) \phi \psi d x \\
& +\int_{\Gamma} \mu(x) \phi \psi d \sigma
\end{aligned}
$$

The proof of the following proposition, in the case $N=3$, can be found in [21, Proposition 9]; the proof for general space dimensions can be easily adapted. Recall that the norm in $H^{1}(\Omega)^{\prime}$ is denoted by $\|\cdot\|_{*}$. 
Proposition 6. Under the assumptions (F1) and (F2) on the function $f$ the energy functional $E \in C^{2}\left(H^{1}(\Omega)\right)$ satisfies the EojasiewiczSimon inequality near every equilibrium point $\phi \in H^{1}(\Omega)$, that is, for every $\phi \in H^{1}(\Omega)$ with $E^{\prime}(\phi)=0$, there exist $\beta_{\phi}>0, \sigma_{\phi}>0$ and $0<\theta_{\phi} \leq 1 / 2$ such that

$$
|E(\phi)-E(\psi)|^{1-\theta_{\phi}} \leq \beta_{\phi}\left\|E^{\prime}(\phi)\right\|_{*}
$$

for all $\psi \in H^{1}(\Omega)$ such that $\|\phi-\psi\|_{H^{1}(\Omega)}<\sigma_{\phi}$. The number $\theta_{\phi}$ is called the Eojasiewicz exponent of $E$ at $\phi$.

2.5. Convergence to equilibrium and decay rate. The following theorem describes the asymptotic behavior of global weak solutions to problem (1).

Theorem 7. Let $u: \mathbf{R}^{+} \rightarrow H^{1}(\Omega)$ be a global, bounded, weak solution of equation (1). Suppose that $f$ satisfies (F1), (F2), and that $\left(g_{1}, g_{2}\right)$ satisfies the growth condition (G2). Then, the solution converges to a single stationary state, which follows from the integrability of $u_{t}$. That is, there exists $\phi \in H^{1}(\Omega)$, solution of the stationary problem:

$$
\begin{cases}-\Delta \phi+f(x, \phi)=0 & \text { in } \Omega, \\ \partial_{\nu} \phi+\mu \phi=0 & \text { on } \Gamma,\end{cases}
$$

such that

$$
\left\|K_{1}^{1 / 2} u_{t}(t)\right\|_{2}+\|u(t)-\phi\|_{H^{1}(\Omega)} \longrightarrow 0 \quad \text { as } t \rightarrow \infty .
$$

From the proof of Theorem 7 and the differential inequality given below (Lemma 15), we deduce in addition that the Łojasiewicz exponent $\theta$ in the Łojasiewicz-Simon inequality determines the decay rate of the solution $u$ to the steady state $\phi$.

Theorem 8. Let $\theta=\theta_{\phi}$ be the Eojasiewicz exponent of $E$ at $\phi$, where $\phi$ is given by Theorem 7 , and let $\delta$ be given by (G2). Then, the following assertions hold:

(i) If $\theta \in(0,(1 / 2))$, then there exists a constant $C>0$ such that

$$
\|u(t)-\phi\|_{2} \leq C(1+t)^{-\xi} \quad \text { for every } t \geq 0,
$$


where

$$
\xi= \begin{cases}\inf \{(\theta /(1-2 \theta)),(\delta / 2)\} & \text { if }\left(g_{1}, g_{2}\right) \neq(0,0) \\ (\theta /(1-2 \theta)) & \text { if }\left(g_{1}, g_{2}\right)=(0,0)\end{cases}
$$

(ii) If $\theta=(1 / 2)$ and $\left(g_{1}, g_{2}\right)=(0,0)$, then there exist constants $C$ and $\kappa>0$ such that

$$
\|u(t)-\phi\|_{2} \leq C e^{-\theta \kappa t}
$$

3. Existence and uniqueness. In this section, we prove the existence and uniqueness of strong/weak solutions of problem (1), that is, we prove Theorem 3. First, we prove the existence and the uniqueness of strong solutions satisfying the properties (T1)-(T4), when the initial data and the source terms are sufficiently smooth. Then we extend the same results to weak solutions by using an approximation argument.

For the convenience of the reader, we recall here explicitly some auxiliary lemmas which will be used in the proof below. We begin with the subsequent simple lemma [20, Lemma 2.1].

Lemma 9. Let $\mathcal{H}$ be a Hilbert space and $T>0$. Suppose that $k \in L_{\mathrm{loc}}^{1}\left(\mathbf{R}^{+}\right)$is nonnegative. Then, for any $v \in L^{2}([0, T] ; \mathcal{H})$, the following holds:

$$
\|(k * v)(t)\|_{\mathcal{H}}^{2} \leq\left(k *\|v\|_{\mathcal{H}}^{2}(1 * k)(t)\right) \quad \text { for almost every } t \in(0, T) .
$$

The second lemma [20, Theorem 2.1] is one key to finding a proper Lyapunov function for problem (1).

Lemma 10. Let $\mathcal{H}$ be a Hilbert space, $T>0$, and $b \in L_{\text {loc }}^{1}\left(\mathbf{R}^{+}\right)$ nonnegative and nonincreasing such that $b * k=1$ in $(0, \infty)$ for some nonnegative kernel $k \in L_{\mathrm{loc}}^{1}\left(\mathbf{R}^{+}\right)$. Suppose that $v \in L^{2}(0, T ; \mathcal{H})$ is such that $b * v \in H^{1}(0, T ; \mathcal{H})$ as well as $b *\|v\|_{\mathcal{H}}^{2} \in W^{1,1}(0, T)$. Then

$$
\begin{aligned}
\left(v(t), \frac{d}{d t}(b * v)(t)\right)_{\mathcal{H}} \geq & \frac{1}{2} \frac{d}{d t}\left(b *\|v\|_{\mathcal{H}}^{2}\right)(t) \\
& +\frac{1}{2} b(t)\|v\|_{\mathcal{H}}^{2} \quad \text { for almost every } t \in(0, T) .
\end{aligned}
$$


Remark 11. (a) Under the same assumptions on kernel $b$, the inequality (14) in Lemma 10 is also satisfied for any function $v \in$ $H^{1}(0, T ; \mathcal{H}),[\mathbf{2 0}$, Remark 2.1].

(b) For the kernels $k$ and $b$ given as in Remark 2 (e), the inequality (14) in Lemma 10 is also satisfied for any function $v \in L^{2}(0, T ; \mathcal{H})$ such that $b * v \in H^{1}(0, T ; \mathcal{H}),[\mathbf{2 0}$, Example 2.1].

Proof of Theorem 3. Existence of strong solution. We transform the problem (1) into an equivalent problem with null initial data. In fact, let us consider the change of variables

$$
v(x, t)=u(x, t)-\phi(x, t)
$$

where

$$
\phi(x, t)=u_{0}(x)+t u_{1}(x) .
$$

Due to this change of variables and the regularity of the initial data we get the following equivalent problem for the variable $v$ :

$$
\begin{cases}K_{1} v_{t t}+K_{2} v_{t}-\Delta v+f(x, v+\phi)=\mathcal{F} & \text { in } \mathbf{R}^{+} \times \Omega \\ \partial_{\nu} v+\mu(x) v+k * v_{t}=\mathcal{G} & \text { on } \mathbf{R}^{+} \times \Gamma \\ v(0)=0, \quad\left(K_{1}\right)^{1 / 2} v_{t}(0)=0 & \end{cases}
$$

Here,

$$
\mathcal{F}=-K_{2} u_{1}+\Delta \phi+g_{1}
$$

and

$$
\mathcal{G}=g_{2}-\left(\partial_{\nu} \phi+\mu(x) \phi+k * u_{1}\right)
$$

We note that if $v$ is a solution of the modified problem $(15)$ in $[0, T]$, then $u=v+\phi$ is a solution of (1) on the same interval.

Since $K_{1} \geq 0$, we first perturb problem (15) by the term $\varepsilon v_{t t}(\varepsilon>0)$ and we apply a Faedo-Galerkin method in order to solve the perturbed problem. Then we shall pass to the limit with $\varepsilon \rightarrow 0$ in the perturbed problem and obtain the solution for problem (15). 
Let $K_{1 \varepsilon}:=K_{1}+\varepsilon$, and consider the perturbed problem:

$$
\begin{cases}K_{1 \varepsilon}(x) v_{\varepsilon t t}+K_{2} v_{\varepsilon t}-\Delta v_{\varepsilon} & \\ \quad+f\left(x, v_{\varepsilon}+\phi\right)=\mathcal{F} & \text { in } \mathbf{R}^{+} \times \Omega, \\ \partial_{\nu} v_{\varepsilon}+\mu(x) v_{\varepsilon}+k * v_{\varepsilon t}=\mathcal{G} & \text { on } \mathbf{R}^{+} \times \Gamma, \\ v_{\varepsilon}(0)=0, \quad\left(K_{1 \varepsilon}\right)^{1 / 2} v_{\varepsilon t}(0)=0 . & \end{cases}
$$

Let $\left(w_{i}\right)_{i \in \mathbf{N}}$ be a total family in $H^{2}(\Omega)$ which is orthonormal in $L^{2}(\Omega)$, and let $V_{m}$ be the subspace of $H^{2}(\Omega)$ which is spanned by the first $m$ vectors $w_{1}, \ldots, w_{m}$. Consider the following weak formulation of an approximated problem, namely, to find a solution

$$
v_{\varepsilon m}(t):=\sum g_{i m}(t) w_{i}
$$

of the ordinary differential equation

$$
\begin{aligned}
&\left(K_{1 \varepsilon} v_{\varepsilon m}^{\prime \prime}(t), w\right)_{2}+\left(K_{2} v_{\varepsilon m}^{\prime}(t), w\right)_{2} \\
&+\left(\nabla v_{\varepsilon m}(t), \nabla w\right)_{2}+\left(f\left(v_{\varepsilon m}(t)+\phi\right), w\right)_{2} \\
&+\left(\mu(x) v_{\varepsilon m}(t), w\right)_{\Gamma} \\
&+\int_{0}^{t} k(t-s)\left(v_{\varepsilon m}^{\prime}(s), w\right)_{\Gamma} d s=(\mathcal{F}, w)_{2}+(\mathcal{G}, w)_{\Gamma} \\
& \text { for every } w \in V_{m} \\
& v_{\varepsilon m}(0)=0, \quad v_{\varepsilon m}^{\prime}(0)=0 .
\end{aligned}
$$

By standard arguments from the theory of ordinary differential equations, one proves the existence and uniqueness of a maximal solution of the integro-differential equation $(17)$ on some interval $\left[0, t_{\varepsilon m}\right]$. We show that this solution can be extended to the whole interval $[0, T]$ by using the first estimate as follows.

First estimate. Taking $w=v_{\varepsilon m}^{\prime}$ in (17), we obtain

$$
\begin{aligned}
\frac{d}{d t}\left(\frac{1}{2}\left\|K_{1 \varepsilon}^{1 / 2} v_{\varepsilon m}^{\prime}\right\|_{2}^{2}+\right. & \frac{1}{2}\left\|\nabla v_{\varepsilon m}\right\|_{2}^{2} \\
& \left.+\int_{\Omega} F\left(x, v_{\varepsilon m}+\phi\right) d x+\frac{1}{2}\left\|\mu^{1 / 2} v_{\varepsilon m}\right\|_{\Gamma}^{2}\right) \\
& +\left\|K_{2}^{1 / 2} v_{\varepsilon m}^{\prime}\right\|_{2}^{2}+\left(k * v_{\varepsilon m}^{\prime}, v_{\varepsilon m}^{\prime}\right)_{\Gamma} \\
= & \left(\mathcal{F}, v_{\varepsilon m}^{\prime}\right)_{2}+\left(\mathcal{G}, v_{\varepsilon m}^{\prime}\right)_{\Gamma} \\
& +\int_{\Omega} f\left(v_{\varepsilon m}+\phi\right) u_{1} d x .
\end{aligned}
$$


Let $w_{\varepsilon m}=k * v_{\varepsilon m}^{\prime}$. We use property (K1) in order to write

$$
v_{\varepsilon m}^{\prime}=\frac{d}{d t}\left([b * k] * v_{\varepsilon m}^{\prime}\right)=\frac{d}{d t}\left(b * w_{\varepsilon m}\right),
$$

which yields

$$
\left(k * v_{\varepsilon m}^{\prime}, v_{\varepsilon m}^{\prime}\right)_{\Gamma}=\left(w_{\varepsilon m}, \frac{d}{d t}\left(b * w_{\varepsilon m}\right)\right)_{\Gamma} .
$$

Then, by Lemma 10,

$$
\left(k * v_{\varepsilon m}^{\prime}, v_{\varepsilon m}^{\prime}\right)_{\Gamma} \geq \frac{1}{2} \frac{d}{d t}\left(b *\left\|w_{\varepsilon m}\right\|_{\Gamma}^{2}\right)(t)+\frac{1}{2} b(t)\left\|w_{\varepsilon m}\right\|_{\Gamma}^{2} .
$$

Using this inequality and the decomposition $b=a+\gamma(1 * a)$, we find

$$
\begin{aligned}
\left(k * v_{\varepsilon m}^{\prime}, v_{\varepsilon m}^{\prime}\right)_{\Gamma} \geq & \frac{1}{2} \frac{d}{d t}\left(a *\left\|w_{\varepsilon m}\right\|_{\Gamma}^{2}\right)(t)+\frac{\gamma}{2}\left(a *\left\|w_{\varepsilon m}\right\|_{\Gamma}^{2}\right)(t) \\
& +\frac{b_{\infty}}{2}\left\|w_{\varepsilon m}\right\|_{\Gamma}^{2},
\end{aligned}
$$

where $b_{\infty}=\lim _{t \rightarrow \infty} b(t)=\gamma\|a\|_{L^{1}\left(\mathbf{R}^{+}\right)}$. Using the last inequality, (18), and the fact that $K_{2} \geq k_{0}>0$, we obtain

$$
\begin{aligned}
& \frac{d}{d t}\left(\frac{1}{2}\left\|K_{1 \varepsilon}^{1 / 2} v_{\varepsilon m}^{\prime}\right\|_{2}^{2}+\frac{1}{2}\left\|\nabla v_{\varepsilon m}\right\|_{2}^{2}\right. \\
& \quad+\int_{\Omega} F\left(x, v_{\varepsilon m}+\phi\right) d x+\frac{1}{2}\left\|\mu^{1 / 2} v_{\varepsilon m}\right\|_{\Gamma}^{2} \\
& \left.\quad+\frac{1}{2} a *\left\|w_{\varepsilon m}\right\|_{\Gamma}^{2}\right)+k_{0}\left\|v_{\varepsilon m}^{\prime}\right\|_{2}^{2}+\frac{b_{\infty}}{2}\left\|w_{\varepsilon m}\right\|_{\Gamma}^{2}+\frac{\gamma}{2} a *\left\|w_{\varepsilon m}\right\|_{\Gamma}^{2} \\
& \leq\left(\mathcal{F}, v_{\varepsilon m}^{\prime}\right)_{2}+\left(\mathcal{G}, v_{\varepsilon m}^{\prime}\right)_{\Gamma}+\int_{\Omega} f\left(v_{\varepsilon m}+\phi\right) u_{1} d x .
\end{aligned}
$$

Integrating $(21)$ over the interval $(0, t)$ and observing that $v_{\varepsilon m}(0)=$ 
$v_{\varepsilon m}^{\prime}(0)=0$, it follows that

$$
\begin{aligned}
\frac{1}{2}\left\|K_{1 \varepsilon}^{1 / 2} v_{\varepsilon m}^{\prime}\right\|_{2}^{2}+ & k_{0} \int_{0}^{t}\left\|v_{\varepsilon m}^{\prime}\right\|_{2}^{2} d s \\
& +\frac{1}{2}\left\|\nabla v_{\varepsilon m}\right\|_{2}^{2}+\int_{\Omega} F\left(x, v_{\varepsilon m}+\phi\right) d x \\
& +\frac{1}{2}\left\|\mu^{1 / 2} v_{\varepsilon m}\right\|_{\Gamma}^{2}+\frac{1}{2} a *\left\|w_{\varepsilon m}\right\|_{\Gamma}^{2} \\
& +\frac{b_{\infty}}{2} \int_{0}^{t}\left\|w_{\varepsilon m}\right\|_{\Gamma}^{2} d s+\frac{\gamma}{2} \int_{0}^{t} a *\left\|w_{\varepsilon m}\right\|_{\Gamma}^{2} d s \\
\leq & \int_{0}^{t}\left(\left(\mathcal{F}, v_{\varepsilon m}^{\prime}\right)_{2}+\left(\mathcal{G}, v_{\varepsilon m}^{\prime}\right)_{\Gamma}\right) d s \\
& +\int_{0}^{t} \int_{\Omega} f\left(v_{\varepsilon m}+\phi\right) u_{1} d x d s+\int_{\Omega} F\left(x, u_{0}\right) d x .
\end{aligned}
$$

Next, we shall estimate some terms in (22). In fact, by (F3), we have

$$
\begin{aligned}
\int_{\Omega} F\left(x, v_{\varepsilon m}+\phi\right) d x & \geq-\frac{\lambda}{2} \int_{\Omega}\left|v_{\varepsilon m}+\phi\right|^{2} d x-C \\
& \geq-C\left\|v_{\varepsilon m}\right\|_{2}^{2}-C .
\end{aligned}
$$

By the Cauchy-Schwarz inequality and since $\mathcal{F} \in L^{2}\left(0, T ; L^{2}(\Omega)\right)$,

$$
\begin{aligned}
\int_{0}^{t}\left(\mathcal{F}, v_{\varepsilon m}^{\prime}\right)_{2} d s & \leq \frac{1}{k_{0}} \int_{0}^{t}\|\mathcal{F}\|_{2}^{2} d s+\frac{k_{0}}{4} \int_{0}^{t}\left\|v_{\varepsilon m}^{\prime}\right\|_{2}^{2} d s \\
& \leq \frac{k_{0}}{4} \int_{0}^{t}\left\|v_{\varepsilon m}^{\prime}\right\|_{2}^{2} d s+C .
\end{aligned}
$$

Moreover, by Lemma 9, (K1) and Young's inequality, we have (25)

$$
\begin{aligned}
\left(\mathcal{G}, v_{\varepsilon m}^{\prime}\right)_{\Gamma}= & \left(\mathcal{G}, \frac{d}{d t} b * w_{\varepsilon m}\right)_{\Gamma}=\left(\mathcal{G}, \frac{d}{d t} a * w_{\varepsilon m}\right)_{\Gamma}+\gamma\left(\mathcal{G}, a * w_{\varepsilon m}\right)_{\Gamma} \\
= & \frac{d}{d t}\left(\mathcal{G}, a * w_{\varepsilon m}\right)_{\Gamma}-\left(\mathcal{G}^{\prime}, a * w_{\varepsilon m}\right)_{\Gamma}+\gamma\left(\mathcal{G}, a * w_{\varepsilon m}\right)_{\Gamma} \\
\leq & \frac{d}{d t}\left(\mathcal{G}, a * w_{\varepsilon m}\right)_{\Gamma}+\|a\|_{L^{1}\left(\mathbf{R}^{+}\right)}\left(\gamma\|\mathcal{G}\|_{\Gamma}^{2}+\gamma^{-1}\left\|\mathcal{G}^{\prime}\right\|_{\Gamma}^{2}\right) \\
& +\frac{\gamma}{4\|a\|_{L^{1}\left(\mathbf{R}^{+}\right)}}\left\|a * w_{\varepsilon m}\right\|_{\Gamma}^{2} \\
\leq & \frac{d}{d t}\left(\mathcal{G}, a * w_{\varepsilon m}\right)_{\Gamma}+d\left(\|\mathcal{G}\|_{\Gamma}^{2}+\left\|\mathcal{G}^{\prime}\right\|_{\Gamma}^{2}\right)+\frac{\gamma}{4} a *\left\|w_{\varepsilon m}\right\|_{\Gamma}^{2},
\end{aligned}
$$


where $d=\|a\|_{L^{1}\left(\mathbf{R}^{+}\right)} \max \left(\gamma, \gamma^{-1}\right)$. Then

(26)

$$
\begin{aligned}
\int_{0}^{t}\left(\mathcal{G}, v_{\varepsilon m}^{\prime}\right)_{\Gamma} d s \leq & \left(\mathcal{G}, a * w_{\varepsilon m}\right)_{\Gamma}+d \int_{0}^{t}\left(\|\mathcal{G}\|_{\Gamma}^{2}+\left\|\mathcal{G}^{\prime}\right\|_{\Gamma}^{2}\right) d s \\
& +\frac{\gamma}{4} \int_{0}^{t} a *\left\|w_{\varepsilon m}\right\|_{\Gamma}^{2} d s \\
\leq & \frac{1}{4} a *\left\|w_{\varepsilon m}\right\|_{\Gamma}^{2}+C\|\mathcal{G}\|_{\Gamma}^{2}+d \int_{0}^{t}\left(\|\mathcal{G}\|_{\Gamma}^{2}+\left\|\mathcal{G}^{\prime}\right\|_{\Gamma}^{2}\right) d s \\
& +\frac{\gamma}{4} \int_{0}^{t} a *\left\|w_{\varepsilon m}\right\|_{\Gamma}^{2} d s \\
\leq & \frac{1}{4} a *\left\|w_{\varepsilon m}\right\|_{\Gamma}^{2}+\frac{\gamma}{4} \int_{0}^{t} a *\left\|w_{\varepsilon m}\right\|_{\Gamma}^{2}+C .
\end{aligned}
$$

Also, by the growth condition (F2), Cauchy-Schwarz inequality and Young's inequality, we have

$$
\begin{aligned}
\int_{0}^{t} \int_{\Omega} f\left(v_{\varepsilon m}+\phi\right) u_{1} d x d s & \leq C \int_{0}^{t} \int_{\Omega}\left(1+\left|v_{\varepsilon m}+\phi\right|^{1+\alpha}\right) u_{1} d x d s \\
& \leq C+C \int_{0}^{t} \int_{\Omega}\left|v_{\varepsilon m}\right|^{1+\alpha} u_{1} d x d s \\
& \leq C+C \int_{0}^{t}\left(\left\|\left|v_{\varepsilon m}\right|^{1+\alpha}\right\|_{2}\left\|u_{1}\right\|_{2}\right) d s \\
& \leq C+C \int_{0}^{t}\left\|v_{\varepsilon m}\right\|_{2(\alpha+1)}^{1+\alpha} d s \\
& \leq C+C \int_{0}^{t}\left\|v_{\varepsilon m}\right\|_{H^{1}(\Omega)}^{1+\alpha} d s \\
& \leq C+C \int_{0}^{t}\left\|v_{\varepsilon m}\right\|_{H^{1}(\Omega)}^{2} d s .
\end{aligned}
$$

Combining (22)-(27), we obtain

$$
\begin{gathered}
\frac{1}{2}\left\|K_{1 \varepsilon}^{1 / 2} v_{\varepsilon m}^{\prime}\right\|_{2}^{2}+\frac{3 k_{0}}{4} \int_{0}^{t}\left\|v_{\varepsilon m}^{\prime}\right\|_{2}^{2} d s+\frac{1}{2}\left\|\nabla v_{\varepsilon m}\right\|_{2}^{2}+\frac{1}{2}\left\|\mu^{1 / 2} v_{\varepsilon m}\right\|_{\Gamma}^{2} \\
+\frac{1}{4} a *\left\|w_{\varepsilon m}\right\|_{\Gamma}^{2}+\frac{b_{\infty}}{2} \int_{0}^{t}\left\|w_{\varepsilon m}\right\|_{\Gamma}^{2} d s
\end{gathered}
$$




$$
\begin{aligned}
& +\frac{\gamma}{4} \int_{0}^{t} a *\left\|w_{\varepsilon m}\right\|_{\Gamma}^{2} d s \\
\leq & C\left(\int_{0}^{t}\left\|v_{\varepsilon m}\right\|_{2}^{2} d s+\int_{0}^{t}\left\|v_{\varepsilon m}\right\|_{H^{1}(\Omega)}^{2} d s+\left\|v_{\varepsilon m}\right\|_{2}^{2}+1\right) .
\end{aligned}
$$

Observe that

$$
\begin{aligned}
C\left\|v_{\varepsilon m}\right\|_{2}^{2}= & C \int_{0}^{t} \frac{d}{d t}\left\|v_{\varepsilon m}(s)\right\|_{2}^{2} d s \leq \frac{C^{2}}{k_{0}} \int_{0}^{t}\left\|v_{\varepsilon m}(s)\right\|_{2}^{2} d s \\
& +\frac{k_{0}}{4} \int_{0}^{t}\left\|v_{\varepsilon m}^{\prime}(s)\right\|_{2}^{2} d s .
\end{aligned}
$$

Using this inequality and (28), we obtain

(29) $\frac{1}{2}\left\|K_{1 \varepsilon}^{1 / 2} v_{\varepsilon m}^{\prime}\right\|_{2}^{2}+\frac{k_{0}}{2} \int_{0}^{t}\left\|v_{\varepsilon m}^{\prime}\right\|_{2}^{2} d s$

$$
\begin{aligned}
& +\frac{1}{2}\left\|\nabla v_{\varepsilon m}\right\|_{2}^{2}+\frac{1}{2}\left\|\mu^{1 / 2} v_{\varepsilon m}\right\|_{\Gamma}^{2} \\
& +\frac{1}{4} a *\left\|w_{\varepsilon m}\right\|_{\Gamma}^{2}+\frac{b_{\infty}}{2} \int_{0}^{t}\left\|w_{\varepsilon m}\right\|_{\Gamma}^{2} d s \\
& +\frac{\gamma}{4} \int_{0}^{t} a *\left\|w_{\varepsilon m}\right\|_{\Gamma}^{2} d s \\
& \leq C \int_{0}^{t}\left\|v_{\varepsilon m}\right\|_{H^{1}(\Omega)}^{2} d s+C .
\end{aligned}
$$

By using this inequality and Gronwall's inequality, we obtain that

$$
\begin{gathered}
\left\|K_{1 \varepsilon}^{1 / 2} v_{\varepsilon m}^{\prime}\right\|_{2}^{2}+\int_{0}^{T}\left\|v_{\varepsilon m}^{\prime}\right\|_{2}^{2} d s+\left\|v_{\varepsilon m}\right\|_{H^{1}(\Omega)}^{2}+a *\left\|w_{\varepsilon m}\right\|_{\Gamma}^{2}+\int_{0}^{T}\left\|w_{\varepsilon m}\right\|_{\Gamma}^{2} d s \\
\leq C_{T},
\end{gathered}
$$

where $C_{T}$ is a positive constant independent of $m, \varepsilon$ and $t$.

Second estimate. Next, we estimate $v_{\varepsilon m}^{\prime \prime}(0)$. Indeed, taking $w=$ $v_{\varepsilon m}^{\prime \prime}(0)$ in $(17)$ and noting that $v_{\varepsilon m}(0)=v_{\varepsilon m}^{\prime}(0)=0$, we obtain

$$
\left\|K_{1 \varepsilon}^{1 / 2} v_{\varepsilon m}^{\prime \prime}(0)\right\|_{2}^{2}+\left(f\left(u_{0}\right)-\mathcal{F}(0), v_{m}^{\prime \prime}(0)\right)_{2}+\left(\mathcal{G}(0), v_{\varepsilon m}^{\prime \prime}(0)\right)_{\Gamma}=0
$$


Using the assumptions on the initial data, we obtain

$$
\left\|K_{1 \varepsilon}^{1 / 2} v_{\varepsilon m}^{\prime \prime}(0)\right\|_{2}=0 .
$$

Also, taking the derivative of (17) with respect to time $t$, taking $w=v_{\varepsilon m}^{\prime \prime}(t)$, and arguing as in the first estimate, we obtain

$$
\begin{aligned}
\frac{d}{d t}\left(\frac{1}{2}\left\|K_{1 \varepsilon}^{1 / 2} v_{\varepsilon m}^{\prime \prime}\right\|_{2}^{2}\right. & \\
& \left.+\frac{1}{2}\left\|\nabla v_{\varepsilon m}^{\prime}\right\|_{2}^{2}+\frac{1}{2}\left\|\mu^{\frac{1}{2}} v_{\varepsilon m}^{\prime}\right\|_{\Gamma}^{2}+\frac{1}{2} a *\left\|z_{\varepsilon m}\right\|_{\Gamma}^{2}\right) \\
& +k_{0}\left\|v_{\varepsilon m}^{\prime \prime}\right\|_{2}^{2}+\frac{b_{\infty}}{2}\left\|z_{\varepsilon m}\right\|_{\Gamma}^{2}+\frac{\gamma}{2} a *\left\|z_{\varepsilon m}\right\|_{\Gamma}^{2} \\
& +\int_{\Omega} f^{\prime}\left(v_{\varepsilon m}+\phi\right)\left(v_{\varepsilon m}^{\prime}+u_{1}\right) v_{\varepsilon m}^{\prime \prime} d x \\
\leq & \left(\mathcal{F}^{\prime}, v_{\varepsilon m}^{\prime \prime}\right)_{2}+\left(\mathcal{G}^{\prime}, v_{\varepsilon m}^{\prime \prime}\right)_{\Gamma}, \quad \text { where } z_{\varepsilon m}=k * v_{\varepsilon m}^{\prime \prime}
\end{aligned}
$$

Integrating this inequality over the interval $(0, t)$ and noticing $v_{\varepsilon m}(0)=$ $v_{\varepsilon m}^{\prime}(0)=\left\|K_{1 \varepsilon}^{1 / 2} v_{\varepsilon m}(0)\right\|_{2}=0$, it follows that

$$
\begin{aligned}
\frac{1}{2}\left\|K_{1 \varepsilon}^{1 / 2} v_{\varepsilon m}^{\prime \prime}\right\|_{2}^{2}+\frac{1}{2} \| & \nabla v_{\varepsilon m}^{\prime} \|_{2}^{2} \\
& +\frac{1}{2}\left\|\mu^{\frac{1}{2}} v_{\varepsilon m}^{\prime}\right\|_{\Gamma}^{2}+\frac{1}{2} a *\left\|z_{\varepsilon m}\right\|_{\Gamma}^{2} \\
& +k_{0} \int_{0}^{t}\left\|v_{\varepsilon m}^{\prime \prime}\right\|_{2}^{2} d s+\frac{b_{\infty}}{2} \int_{0}^{t}\left\|z_{\varepsilon m}\right\|_{\Gamma}^{2} d s \\
& +\frac{\gamma}{2} \int_{0}^{t} a *\left\|z_{\varepsilon m}\right\|_{\Gamma}^{2} d s \\
\leq & -\int_{0}^{t} \int_{\Omega} f^{\prime}\left(v_{\varepsilon m}+\phi\right)\left(v_{\varepsilon m}^{\prime}+u_{1}\right) v_{\varepsilon m}^{\prime \prime} d x d s \\
& +\int_{0}^{t}\left(\left(\mathcal{F}^{\prime}, v_{\varepsilon m}^{\prime \prime}\right)_{2}+\left(\mathcal{G}^{\prime}, v_{\varepsilon m}^{\prime \prime}\right)_{\Gamma}\right) d s .
\end{aligned}
$$

Next, we shall estimate the nonlinear terms of (33). For this, by using 
Hölder's inequality and the first estimate, we obtain (for $N \geq 3$ )

$$
\begin{aligned}
& \int_{0}^{t} \int_{\Omega} f^{\prime}\left(v_{\varepsilon m}+\phi\right)\left(v_{\varepsilon m}^{\prime}+u_{1}\right) v_{\varepsilon m}^{\prime \prime} d x d s \\
& \quad \leq C \int_{0}^{t} \int_{\Omega}\left(1+\left|v_{\varepsilon m}+\phi\right|^{\alpha}\right)\left(v_{\varepsilon m}^{\prime}+u_{1}\right) v_{\varepsilon m}^{\prime \prime} d x d s \\
& \quad \leq C \int_{0}^{t}\left(\left\|1+\left|v_{\varepsilon m}+\phi\right|^{\alpha}\right\|_{N}\left\|v_{\varepsilon m}^{\prime}+u_{1}\right\|_{2 N /(N-2)}\left\|v_{\varepsilon m}^{\prime \prime}\right\|_{2}\right) d s \\
& \quad \leq C \int_{0}^{t}\left(\left(1+\left\|v_{\varepsilon m}+\phi\right\|_{N \alpha}^{\alpha}\right)\left\|v_{\varepsilon m}^{\prime}+u_{1}\right\|_{2 N /(N-2)}\left\|v_{\varepsilon m}^{\prime \prime}\right\|_{2}\right) d s \\
& \quad \leq \frac{k_{0}}{4} \int_{0}^{t}\left\|v_{\varepsilon m}^{\prime \prime}\right\|_{2}^{2} d s+C \int_{0}^{t}\left\|v_{\varepsilon m}^{\prime}+u_{1}\right\|_{2 N /(N-2)}^{2} d s \\
& \quad \leq \frac{k_{0}}{4} \int_{0}^{t}\left\|v_{\varepsilon m}^{\prime \prime}\right\|_{2}^{2} d s+C \int_{0}^{t}\left\|v_{\varepsilon m}^{\prime}\right\|_{H^{1}(\Omega)}^{2} d s+C .
\end{aligned}
$$

The inequality (34) still holds when $N \leq 2$ by using the boundedness of $v_{\varepsilon m}$ in $H^{1}(\Omega)$ (first estimate) and the fact that:

- $H^{1}(\Omega) \hookrightarrow C(\bar{\Omega})$ in the one-dimensional case and

- $H^{1}(\Omega) \hookrightarrow L^{p}(\Omega)$, for all $p \in[1, \infty[$, if $N=2$.

Again, by the Cauchy-Schwarz inequality and since $\mathcal{F}^{\prime} \in L^{2}\left([0, T] ; L^{2}(\Omega)\right)$ :

$$
\int_{0}^{t}\left(\mathcal{F}^{\prime}, v_{\varepsilon m}^{\prime \prime}\right)_{2} d s \leq \frac{k_{0}}{4} \int_{0}^{t}\left\|v_{\varepsilon m}^{\prime \prime}\right\|_{2}^{2} d s+C .
$$

Moreover, similarly as in (26),

$$
\int_{0}^{t}\left(\mathcal{G}^{\prime}, v_{\varepsilon m}^{\prime \prime}\right)_{\Gamma} d s \leq \frac{1}{4} a *\left\|z_{\varepsilon m}\right\|_{\Gamma}^{2}+\frac{\gamma}{4} \int_{0}^{t} a *\left\|z_{\varepsilon m}\right\|_{\Gamma}^{2} d s+C .
$$

Combining (33)-(36) and applying Gronwall's inequality, we obtain

$$
\begin{aligned}
\left\|K_{1 \varepsilon}^{\frac{1}{2}} v_{\varepsilon m}^{\prime \prime}\right\|_{2}^{2}+\int_{0}^{T}\left\|v_{\varepsilon m}^{\prime \prime}\right\|_{2}^{2} d s+\left\|v_{\varepsilon m}^{\prime}\right\|_{H^{1}(\Omega)}^{2}+a *\left\|z_{\varepsilon m}\right\|_{\Gamma}^{2} & \\
& +\int_{0}^{T}\left\|z_{\varepsilon m}\right\|_{\Gamma}^{2} d s \leq C_{T},
\end{aligned}
$$

where $C_{T}$ is a positive constant independent of $m, \varepsilon$ and $t$. 
Passing to the limit. Using estimates (30) and (37) and passing to the limit (first $m \rightarrow \infty$, and then $\varepsilon \rightarrow 0$ ), we see that there exists a strong solution $u \in W_{\mathrm{loc}}^{1, \infty}\left(\mathbf{R}^{+} ; H^{1}(\Omega)\right) \cap W_{\mathrm{loc}}^{2,2}\left(\mathbf{R}^{+} ; L^{2}(\Omega)\right)$, $K_{1}^{1 / 2} u_{t} \in W_{\text {loc }}^{1, \infty}\left(\mathbf{R}^{+} ; L^{2}(\Omega)\right)$. In addition, $u$ satisfies, for every $t \geq 0$, the nonhomogeneous Neumann problem:

$$
\begin{cases}-\Delta u=-K_{1} u_{t t}-K_{2} u_{t}-f(x, u)+g_{1} & \text { in } L^{2}(\Omega), \\ \partial_{\nu} u=-\mu u-k * u_{t}+g_{2} & \text { in } H^{1 / 2}(\Gamma) .\end{cases}
$$

The theory of elliptic problems gives us $u \in L_{\mathrm{loc}}^{\infty}\left(\mathbf{R}^{+} ; H^{2}(\Omega)\right)$.

Boundedness and energy estimate for strong solutions. Now, let $u$ be a global strong solution of (1), and let $v=k * u_{t}$. We take the inner product of equation (1) with $u_{t}$ in order to find that

$$
\begin{aligned}
\frac{d}{d t}\left(\frac{1}{2}\left\|K_{1}^{1 / 2} u_{t}\right\|_{2}^{2}+E(u)\right)+\left(K_{2}(x) u_{t}, u_{t}\right)_{2} & +\left(v, u_{t}\right)_{\Gamma} \\
& =\left(g_{1}, u_{t}\right)_{2}+\left(g_{2}, u_{t}\right)_{\Gamma}
\end{aligned}
$$

Using that $K_{2}$ is strictly positive and the Cauchy-Schwarz inequality, we find

$$
\begin{array}{r}
\frac{d}{d t}\left(\frac{1}{2}\left\|K_{1}^{1 / 2} u_{t}\right\|_{2}^{2}+E(u)+\frac{1}{2 k_{0}} \int_{t}^{\infty}\left\|g_{1}(s)\right\|_{2}^{2} d s\right)+\left(k * u_{t}, u_{t}\right)_{\Gamma} \\
\leq-\frac{k_{0}}{2}\left\|u_{t}\right\|_{2}^{2}+\left(g_{2}, u_{t}\right)_{\Gamma}
\end{array}
$$

Using the regularity of the strong solution, Remark 11 and arguing as in (20), we obtain

$$
\left(k * u_{t}, u_{t}\right)_{\Gamma} \geq \frac{1}{2} \frac{d}{d t} a *\|v\|_{\Gamma}^{2}+\frac{b_{\infty}}{2}\|v\|_{\Gamma}^{2}+\frac{\gamma}{2} a *\|v\|_{\Gamma}^{2}
$$

where $b_{\infty}=\lim _{t \rightarrow \infty} b(t)=\gamma\|a\|_{L^{1}\left(\mathbf{R}^{+}\right)}$. Moreover, by Lemma 9 and by Young's inequality, we have (as in (25))

$$
\begin{gathered}
\left(g_{2}, u_{t}\right)_{\Gamma} \leq \frac{d}{d t}\left(g_{2}, a * v\right)_{\Gamma}+d\left(\left\|g_{2}\right\|_{\Gamma}^{2}+\left\|g_{2}^{\prime}\right\|_{\Gamma}^{2}\right)+\frac{\gamma}{4} a *\|v\|_{\Gamma}^{2} \\
(t>0) .
\end{gathered}
$$


Combining (39), (40) and (41), one obtains (12) for every strong solution. In addition, from the condition (F2) we have

$$
\int_{\Omega}\left|F\left(x, u_{0}\right)\right| \leq C\left(1+\left\|u_{0}\right\|_{H^{1}}^{\alpha+2}\right),
$$

where $C \geq 0$ is a constant depending only on the constants from condition (F2) (including the norm $\|f(\cdot, 0)\|_{L^{\infty}}$ ) and the constant of the embedding $H^{1}(\Omega) \hookrightarrow L^{\alpha+2}(\Omega)$. It follows from this inequality and the definition of $G$ that there exists a constant $C_{1} \geq 0$ which is independent of the initial data such that

$$
G(0) \leq C_{1}\left(1+\left\|K_{1}^{1 / 2} u_{1}\right\|_{L^{2}}^{2}+\left\|u_{0}\right\|_{H^{1}}^{\alpha+2} .\right.
$$

On the other hand, by using condition (F3), the definition of $G$, the boundedness of $g_{2}$ with values in $L^{2}(\Gamma)$, and the following estimates given by Lemma 9 , that is,

$$
\left(g_{2}, a * v\right)_{\Gamma} \leq\|a\|_{L^{1}\left(\mathbf{R}^{+}\right)}\left\|g_{2}\right\|_{\Gamma}^{2}+\frac{1}{4} a *\|v\|_{\Gamma}^{2},
$$

one easily shows that there exists a positive constant $C_{2}$ depending on $\lambda$ and $\lambda_{1}$, and a positive constant $C_{3}$ depending on $f, g_{2}$ and the measure of $\Omega$ such that, for every $t \geq 0$,

$$
\|u(t)\|_{H^{1}(\Omega)}^{2}+\left\|K_{1}^{1 / 2} u_{t}(t)\right\|_{2}^{2} \leq C_{2} G(t)+C_{3} .
$$

We combine (12), (42) and (44) to obtain the a priori estimate

$$
\begin{aligned}
\|u(t)\|_{H^{1}(\Omega)}^{2}+\| K_{1}^{1 / 2} & u_{t}(t) \|_{2}^{2} \\
& +\int_{0}^{t}\left\|u_{t}(s)\right\|_{2}^{2} d s+\int_{0}^{t}\|v(s)\|_{\Gamma}^{2} d s \\
\leq & C_{4}\left(1+\left\|K_{1}^{1 / 2} u_{1}\right\|_{L^{2}}^{2}+\left\|u_{0}\right\|_{H^{1}}^{\mu+2}\right) \quad(t \geq 0),
\end{aligned}
$$

where $C_{4} \geq 0$ depends only on the constants $C_{1}, C_{2}, C_{3}$ and on $g_{1}$, but is independent of the initial data. This a priori estimate gives the boundedness of strong solutions in $H^{1}(\Omega)$.

Uniqueness and continuous dependence. Next we show the continuous dependence of strong solutions on the initial data. Let $u^{p}(p=1,2)$ be 
two strong solutions of (1), corresponding to the initial data $\left(u_{0}^{p}, u_{1}^{p}\right)$ and the forcing terms $\left(g_{1}^{p}, g_{2}^{p}\right)(p=1,2)$. Setting $\bar{u}=u^{1}-u^{2}$, $g_{1}=g_{1}^{1}-g_{1}^{2}$ and $g_{2}=g_{2}^{1}-g_{2}^{2}$, one has

$$
\begin{cases}K_{1} \bar{u}_{t t}+K_{2} \bar{u}_{t}-\Delta \bar{u}+f\left(x, u^{1}\right)-f\left(x, u^{2}\right)=g_{1} & \text { in } \mathbf{R}^{+} \times \Omega \\ \partial_{\nu} \bar{u}+\mu \bar{u}+k * \bar{u}_{t}=g_{2} & \text { on } \mathbf{R}^{+} \times \Gamma \\ \bar{u}(0)=u_{0}^{1}-u_{0}^{2}, \sqrt{K_{1}} \bar{u}_{t}(0)=\sqrt{K_{1}} u_{1}^{1}-\sqrt{K_{1}} u_{1}^{2} & \end{cases}
$$

Let $h=k * \bar{u}_{t}$. We multiply equation (46) with $\bar{u}_{t}$ and integrate over $\Omega$, in order to find that

$$
\begin{aligned}
\frac{d}{d t}\left(\frac{1}{2}\left\|K_{1}^{\frac{1}{2}} \bar{u}_{t}\right\|_{2}^{2}+\right. & \frac{1}{2}\|\nabla \bar{u}\|_{2}^{2}+\frac{1}{2}\left\|\mu^{1 / 2} \bar{u}\right\|_{\Gamma}^{2} \\
& +\frac{1}{2} a *\|h\|_{\Gamma}^{2}-\left(g_{2}, a * h\right)_{\Gamma}+\frac{1}{2 k_{0}} \int_{t}^{\infty}\left\|g_{1}(s)\right\|_{2}^{2} d s \\
& \left.+d \int_{t}^{\infty}\left(\left\|g_{2}(s)\right\|_{\Gamma}^{2}+\left\|g_{2}^{\prime}(s)\right\|_{\Gamma}^{2}\right) d s\right) \\
& +\int_{\Omega}\left(f\left(u^{1}\right)-f\left(u^{2}\right)\right)\left(u_{t}^{1}-u_{t}^{2}\right) d x \\
& +\frac{k_{0}}{2}\left\|\bar{u}_{t}\right\|_{2}^{2}+\frac{b_{\infty}}{2}\|h\|_{\Gamma}^{2}+\frac{\gamma}{4} a *\|h\|_{\Gamma}^{2} \\
\leq & 0,
\end{aligned}
$$

where we have used (40) and (41), when $(u, v)$ are replaced by $(\bar{u}, h)$.

Integrating this inequality over $(0, t)$, using $(43)$, and the fact that the Nemytskii operator generated by $f$ is locally Lipschitz continuous from $H^{1}(\Omega)$ into $L^{2}(\Omega)$ (note that $u^{1}$ and $u^{2}$ are bounded in $C\left(\mathbf{R}^{+}, H^{1}(\Omega)\right.$ ) by $(45))$, we obtain

$$
\begin{aligned}
\frac{1}{2}\left\|K_{1}^{1 / 2} \bar{u}_{t}\right\|_{2}^{2}+\frac{1}{2}\|\nabla \bar{u}\|_{2}^{2}+1 / 2\left\|\mu^{1 / 2} \bar{u}\right\|_{\Gamma}^{2} \\
\quad+\frac{1}{4} a *\|h\|_{\Gamma}^{2}+\frac{k_{0}}{4} \int_{0}^{t}\left\|\bar{u}_{t}\right\|_{2}^{2} d s+\frac{b_{\infty}}{2} \int_{0}^{t}\|h\|_{\Gamma}^{2} d s \\
\quad+\frac{\gamma}{4} \int_{0}^{t} a *\|h\|_{\Gamma}^{2} d s+\frac{1}{2 k_{0}} \int_{t}^{\infty}\left\|g_{1}(s)\right\|_{2}^{2} d s \\
\quad+d \int_{t}^{\infty}\left(\left\|g_{2}(s)\right\|_{\Gamma}^{2}+\left\|g_{2}^{\prime}(s)\right\|_{\Gamma}^{2}\right) d s
\end{aligned}
$$




$$
\begin{aligned}
\leq & C \int_{0}^{t}\|\bar{u}(s)\|_{H^{1}(\Omega)}^{2} d s+\frac{1}{2 k_{0}} \int_{0}^{\infty}\left\|g_{1}(s)\right\|_{2}^{2} d s \\
& +d \int_{0}^{\infty}\left(\left\|g_{2}(s)\right\|_{\Gamma}^{2}+\left\|g_{2}^{\prime}(s)\right\|_{\Gamma}^{2}\right) d s \\
& +C\left(\left\|K_{1}^{1 / 2} \bar{u}_{t}(0)\right\|_{2}^{2}+\|\bar{u}(0)\|_{H^{1}}^{2}\right) .
\end{aligned}
$$

From this inequality and Gronwall's lemma we infer that, for every $t \geq 0$,

$$
\begin{aligned}
\left\|K_{1}^{1 / 2} \bar{u}_{t}(t)\right\|_{2}^{2}+\|\bar{u}(t)\|_{H^{1}(\Omega)}^{2} & \\
& +\int_{0}^{t}\left\|\bar{u}_{t}(s)\right\|_{2}^{2} d s+\int_{0}^{t}\|h\|_{\Gamma}^{2} d s \\
\leq & C e^{C t}\left(\int_{0}^{t}\left\|g_{1}(s)\right\|_{2}^{2} d s+\int_{t}^{\infty}\left(\left\|g_{2}(s)\right\|_{\Gamma}^{2}+\left\|g_{2}^{\prime}(s)\right\|_{\Gamma}^{2}\right) d s\right. \\
& \left.+\left\|K_{1}^{1 / 2} \bar{u}_{t}(0)\right\|_{2}^{2}+\|\bar{u}(0)\|_{H^{1}}^{2}\right) .
\end{aligned}
$$

The continuous dependence of strong solutions on initial data, and the uniqueness of strong solutions are both an immediate consequence of this inequality.

Existence and uniqueness of weak solutions. Let $\left(u_{0}, u_{1}\right) \in \bar{D}$ and $\left(g_{1}, g_{2}\right) \in L_{\text {loc }}^{2}\left(\mathbf{R}^{+} ; L^{2}(\Omega)\right) \times W^{1,2}\left(\mathbf{R}^{+} ; L^{2}(\Gamma)\right)$. Then there exists a sequence $\left(\left(u_{0}^{p}, u_{1}^{p}\right)\right)_{p} \subseteq H^{2}(\Omega) \times H^{3 / 2}(\Omega)$ satisfying the compatibility condition $(9)$, and a sequence $\left(\left(g_{1}^{p}, g_{2}^{p}\right)\right)_{p}$ satisfying the regularity properties (8), such that

$$
\begin{array}{ll}
\left(u_{0}^{p}, u_{1}^{p}\right) \longrightarrow\left(u_{0}, u_{1}\right) & \text { in } H^{1}(\Omega) \times L^{2}(\Omega), \quad \text { and } \\
\left(g_{1}^{p}, g_{2}^{p}\right) \longrightarrow\left(g_{1}, g_{2}\right) & \text { in } L_{\mathrm{loc}}^{2}\left(\mathbf{R}^{+} ; L^{2}(\Omega)\right) \times W^{1,2}\left(\mathbf{R}^{+} ; L^{2}(\Gamma)\right) .
\end{array}
$$

Then, for each $p \in \mathbf{N}$, there exists a unique strong solution $u^{p}$ to the problem (1). By estimate (45), we have

$$
\begin{aligned}
u^{p} & \text { is uniformly bounded in } C_{b}\left(\mathbf{R}^{+} ; H^{1}(\Omega)\right), \\
u_{t}^{p} & \text { is uniformly bounded in } L^{2}\left(\mathbf{R}^{+} ; L^{2}(\Omega)\right), \\
K_{1}^{1 / 2} u_{t}^{p} & \text { is uniformly bounded in } C_{b}\left(\mathbf{R}^{+} ; L^{2}(\Omega)\right), \\
k * u_{t}^{p} & \text { is uniformly bounded in } L^{2}\left(\mathbf{R}^{+} ; L^{2}(\Gamma)\right) .
\end{aligned}
$$


Moreover, by the estimate (48) we have

$u^{p}$ is a Cauchy sequence in $C\left(\mathbf{R}^{+} ; H^{1}(\Omega)\right)$,

$u_{t}^{p} \quad$ is a Cauchy sequence in $L_{\mathrm{loc}}^{2}\left(\mathbf{R}^{+} ; L^{2}(\Omega)\right)$,

$K_{1}^{1 / 2} u_{t}^{p} \quad$ is a Cauchy sequence in $C\left(\mathbf{R}^{+} ; L^{2}(\Omega)\right)$,

$k * u_{t}^{p} \quad$ is a Cauchy sequence in $L_{\mathrm{loc}}^{2}\left(\mathbf{R}^{+} ; L^{2}(\Gamma)\right)$.

The convergences given by (49) and (50) are sufficient to obtain a weak solution $u$ to problem (1) as the strong limit of the above sequence of strong solutions, that is,

$$
\begin{aligned}
u^{p} \longrightarrow u & \text { in } C\left(\mathbf{R}^{+} ; H^{1}(\Omega)\right), \\
u_{t}^{p} \longrightarrow u_{t} & \text { in } L_{\mathrm{loc}}^{2}\left(\mathbf{R}^{+} ; L^{2}(\Omega)\right), \\
K_{1}^{1 / 2} u_{t}^{p} \longrightarrow K_{1}^{1 / 2} u_{t} & \text { in } C\left(\mathbf{R}^{+} ; L^{2}(\Omega)\right), \\
k * u_{t}^{p} \longrightarrow v=\frac{d}{d t}\left(k *\left(u-u_{0}\right)\right) & \text { in } L_{\mathrm{loc}}^{2}\left(\mathbf{R}^{+} ; L^{2}(\Gamma)\right) .
\end{aligned}
$$

However, from (51), one easily sees that the energy inequality (12), the estimate (45) and the a priori estimate (48) remain true for any weak solution, respectively any pair of weak solutions. The uniqueness of weak solutions is again an immediate consequence of the a priori estimate (48). From the estimate (45) we obtain that every weak solution is bounded (property (T1)). Moreover, by (12), the boundedness of $u$ in $H^{1}(\Omega)$, the continuity of $E$, and (43), the energy function $G$ is decreasing and bounded from below, and therefore

$$
\lim _{t \rightarrow \infty} G(t)=\inf _{t \geq 0} G(t)=G_{\infty} \text { exists. }
$$

From this and the energy inequality (12), we obtain (T2). Finally, in order to prove the variational equality (T4) we note first that this equality is satisfied pointwise (in time) for any strong solution. However, by using again that weak solutions are locally uniform limits of strong solutions, one sees that this equality remains valid for all weak solutions.

4. Compact range of global and bounded solutions. In this section we obtain a compactness result which generalizes the previous 
results in $[\mathbf{1 3}]$ to the case of dynamical boundary conditions. In order to prove Theorem 5, let us list two lemmas for which we need the following notation. Let $X$ be a (real) Banach space equipped with the norm $\|\cdot\|_{X}$, and let $S^{2}\left(\mathbf{R}^{+} ; X\right)$ be the Stepanov space defined by

$$
S^{2}\left(\mathbf{R}^{+} ; X\right)=\left\{g \in L_{\mathrm{loc}}^{2}\left(\mathbf{R}^{+} ; X\right), \sup _{t \in \mathbf{R}^{+}} \int_{t}^{t+1}\|g(s)\|_{X}^{2} d s<\infty\right\} .
$$

For any $h>0, t \geq 0$ and any $g \in S^{2}\left(\mathbf{R}^{+} ; X\right)$ we denote by $g^{h}(t)$ the difference $g(t+h)-g(t)$ and we say that $g$ is $S^{1}$-uniformly continuous with values in $X$ if

$$
\sup _{t \in \mathbf{R}^{+}} \int_{t}^{t+1}\left\|g^{h}(s)\right\|_{X}^{2} d s \longrightarrow 0 \quad \text { as } h \rightarrow 0 .
$$

Lemma 12 [5]. Let $u$ be a global bounded weak solution of (1). Assume that $f$ satisfies (F2) and that $g_{1}$ satisfies (G2). Then the source term $H(t)=g_{1}(t)-f(t, u)$ is $S^{1}$-uniformly continuous in $L^{2}(\Omega)$ and $H \in S^{2}\left(\mathbf{R}^{+}, L^{2}(\Omega)\right)$.

Lemma 13 [13]. Let $X$ and $Y$ be two Banach spaces endowed respectively with the norms $\|\cdot\|_{X}$ and $\|\cdot\|_{Y}$. Assume that $X$ is compactly embedded into $Y$. Then:

(a) If $u: \mathbf{R}^{+} \rightarrow Y$ is uniformly continuous and

$$
\sup _{\substack{t \geq 0 \\ \delta \in[0,1]}}\left\|\int_{t}^{t+\delta} u(s) d s\right\|_{X}<\infty
$$

then $\bigcup_{t \geq 0}\{u(t)\}$ is precompact in $Y$.

(b) If $u \in C^{1}\left(\mathbf{R}^{+}, Y\right)$ is bounded with values in $X$, and if $u^{\prime}$ is uniformly continuous with values in $Y$, then $\bigcup_{t \geq 0}\left\{u^{\prime}(t)\right\}$ is precompact in $Y$.

Proof of Theorem 5. We proceed in two steps.

Step 1 . We first show that the function $\left(u(t), K_{1}^{1 / 2} u_{t}(t)\right)$ is uniformly continuous with values in $H^{1}(\Omega) \times L^{2}(\Omega)$. For all $t \geq 0, h \geq 0$, 
we let $u^{h}(t)=u(t+h)-u(t)$. Since $u_{t} \in L^{2}\left(\mathbf{R}^{+} ; L^{2}(\Omega)\right)$ and $g_{1}-f(\cdot, u) \in S^{2}\left(\mathbf{R}^{+}, L^{2}(\Omega)\right)$, we have

$$
\sup _{t \geq 0} \int_{t}^{t+1}\left\|K_{1} u_{t t}-\Delta u\right\|_{2} d s \leq C .
$$

From this estimate and (1), we easily deduce the inequality

$$
\begin{aligned}
& \int_{t}^{t+1}\left\|u^{h}(s)\right\|_{H^{1}(\Omega)}^{2} d s \\
\leq & C\left\{\int_{t}^{t+1}\left(\mu u^{h}(s), \mu u^{h}(s)\right)_{\Gamma}+\left(\nabla u^{h}(s), \nabla u^{h}(s)\right)_{2} d s\right\} \\
\leq & C\left\{\int_{t}^{t+1}\left(\mu u^{h}(s)+\partial_{\nu} u^{h}(s), \mu u^{h}(s)\right)_{\Gamma}+\left(-\Delta u^{h}(s), u^{h}(s)\right)_{2} d s\right\} \\
\leq & C\left\{\int_{t}^{t+1}\left\|K_{1}^{1 / 2} u_{t}^{h}(s)\right\|_{2}^{2} d s+\left\|K_{1} u_{t}^{h}(t)\right\|_{2}\left\|u^{h}(t)\right\|_{2}\right. \\
& +\left\|K_{1} u_{t}^{h}(t+1)\right\|_{2}\left\|u^{h}(t+1)\right\|_{2} \\
& \left.+\int_{t}^{t+1}\left\|g_{2}^{h}-v^{h}\right\|_{\Gamma}^{2} d s+\sup _{[t, t+1]}\left\|u^{h}\right\|_{2}\right\} \\
\leq & C\left\{\int_{t}^{t+1}\left\|K_{1}^{1 / 2} u_{t}^{h}(s)\right\|_{2}^{2} d s+\int_{t}^{t+1}\left\|g_{2}^{h}-v^{h}\right\|_{\Gamma}^{2} d s+C_{3} \sup _{[t, t+1]}\left\|u^{h}\right\|_{2}\right\} .
\end{aligned}
$$

Since $u_{t} \in L^{2}\left(\mathbf{R}^{+} ; L^{2}(\Omega)\right), u$ is uniformly continuous from $\mathbf{R}^{+}$into $L^{2}(\Omega)$. Using this and the last inequality, we obtain

$$
\begin{aligned}
\int_{t}^{t+1}\left\|u^{h}(s)\right\|_{H^{1}(\Omega)}^{2} d s \leq & C\left\{\int_{t}^{t+1}\left\|K_{1}^{1 / 2} u_{t}^{h}(s)\right\|_{2}^{2} d s+\int_{t}^{t+1}\left\|g_{2}^{h}-v^{h}\right\|_{\Gamma}^{2} d s\right\} \\
& +\phi_{1}(h),
\end{aligned}
$$

where $\phi_{1}(h) \rightarrow 0$ as $h \rightarrow 0$. Moreover, since $u_{t} \in L^{2}\left(\mathbf{R}^{+} ; L^{2}(\Omega)\right)$ and since the left-shift semigroup on the space $L^{2}\left(\mathbf{R}^{+} ; L^{2}(\Omega)\right)$ is strongly continuous, then we have

$$
\int_{t}^{t+1}\left\|K_{1}^{1 / 2} u_{t}^{h}(s)\right\|_{2}^{2} d s \longrightarrow 0 \quad \text { as } h \rightarrow 0 .
$$


Similarly, since $g_{2}, v \in L^{2}\left(\mathbf{R}^{+} ; L^{2}(\Gamma)\right)$,

$$
\int_{t}^{t+1}\left\|g_{2}^{h}-v^{h}\right\|_{\Gamma}^{2} d s \longrightarrow 0 \quad \text { as } h \rightarrow 0
$$

By using the last two limits and the inequality (53), we obtain

$$
\int_{t}^{t+1}\left\|u^{h}(s)\right\|_{H^{1}(\Omega)}^{2} d s \leq \phi_{2}(h),
$$

where $\phi_{2}(h) \rightarrow 0$ as $h \rightarrow 0$. Now we introduce

$$
V_{h}(t)=\frac{1}{2}\left(\left\|K_{1}^{1 / 2} u_{t}^{h}(t)\right\|_{2}^{2}+\left\|\nabla u^{h}(t)\right\|_{2}^{2}+\left\|\mu^{1 / 2} u^{h}(t)\right\|_{\Gamma}^{2}+a *\left\|v^{h}\right\|_{\Gamma}^{2}(t)\right) .
$$

Since $a *\|v\|_{\Gamma}^{2} \in L^{1}\left(\mathbf{R}^{+}\right)$,

$$
\int_{t}^{t+1} a *\left\|v^{h}\right\|_{\Gamma}^{2}(s) d s \longrightarrow 0 \quad \text { as } h \rightarrow 0 .
$$

Combining (54), (56) and (57), we obtain

$$
\int_{t}^{t+1} V_{h}(\theta) d \theta \leq \phi_{3}(h), \quad \text { where } \phi_{3}(h) \longrightarrow 0 \quad \text { as } h \rightarrow 0 .
$$

On the other hand, for a strong solution $u$, by taking the derivative of $V_{h}(t)$ with respect to $t$, and by using (1) and (40), we obtain (59)

$$
\begin{aligned}
\frac{d}{d t} V_{h}(t) \leq & \left(g_{1}^{h}-f^{h}(x, u), u_{t}^{h}\right)_{2}+\left(g_{2}^{h}, u_{t}^{h}\right)_{\Gamma} \\
& -\left(\left(K_{2} u_{t}^{h}, u_{t}^{h}\right)+\frac{\gamma}{2} a *\left\|v^{h}\right\|_{\Gamma}^{2}+\frac{1}{2} a *\left\|v^{h}\right\|_{\Gamma}^{2}+\frac{b_{\infty}}{2}\left\|v^{h}\right\|_{\Gamma}^{2}\right) \\
\leq & \left(g_{1}^{h}-f^{h}(x, u), u_{t}^{h}\right)_{2}+\left(g_{2}^{h}, u_{t}^{h}\right)_{\Gamma} .
\end{aligned}
$$

Integrating (59) over $[\theta, t+1]$ with $\theta \in[t, t+1]$, using Lemma 12, the fact that $u(t)$ is bounded in $H^{1}(\Omega), u_{t} \in L^{2}\left(\mathbf{R}^{+} ; L^{2}(\Omega)\right)$, and 
$g_{2}^{\prime} \in L^{2}\left(\mathbf{R}^{+} ; L^{2}(\Gamma)\right)$, we deduce that, for any $t \geq 0$ :

(60)

$$
\begin{aligned}
V_{h}(t+1)-V_{h}(\theta) \leq & C \int_{t}^{t+1}\left(\left\|\left(g_{1}-f(x, u)\right)^{h}(s)\right\|_{2}^{2}+\left\|u_{t}^{h}(s)\right\|_{2}^{2}\right) d s \\
& +\int_{\theta}^{t+1}\left(g_{2}^{h}, u_{t}^{h}\right)_{\Gamma}(s) d s \\
\leq & C \int_{t}^{t+1}\left(\left\|\left(g_{1}-f(x, u)\right)^{h}(s)\right\|_{2}^{2}+\left\|u_{t}^{h}(s)\right\|_{2}^{2}\right) d s \\
& -\int_{\theta}^{t+1}\left(\left(g_{2}^{\prime}\right)^{h}, u^{h}\right)_{\Gamma}(s) d s \\
& +C \sup _{[t, t+1]}\left\|g_{2}^{h}(s)\right\|_{\Gamma} \\
\leq & C \int_{t}^{t+1}\left(\left\|\left(g_{1}-f(x, u)\right)^{h}(s)\right\|_{2}^{2}+\left\|u_{t}^{h}(s)\right\|_{2}^{2}\right) d s \\
& +C \int_{t}^{t+1}\left\|\left(g_{2}^{\prime}\right)^{h}(s)\right\|_{\Gamma}^{2} d s \\
& +C \sup _{[t, t+1]}\left\|g_{2}^{h}(s)\right\|_{\Gamma} \\
\leq & \phi_{4}(h), \quad \text { where } \phi_{4}(h) \longrightarrow 0 \text { as } h \rightarrow 0 .
\end{aligned}
$$

By an approximation argument, inequality (60) still holds for all weak solutions. Then, by integrating (60) over $[t, t+1]$ with respect to $\theta$ and by using (58), we obtain

$$
V_{h}(t+1) \leq \phi_{4}(h)+\int_{t}^{t+1} V_{h}(\theta) d \theta \leq \phi_{5}(h),
$$

which tends to 0 as $h \rightarrow 0$. This concludes the proof of Step 1 .

Step 2. We show that $\left(u(t), K_{1}^{1 / 2} u_{t}(t)\right)$ is relatively compact in $H^{1}(\Omega) \times L^{2}(\Omega)$. By applying Lemma 13 (b) with $Y=L^{2}(\Omega)$ and $X=H^{1}(\Omega)$, we obtain immediately that $\bigcup_{t \geq 0}\left\{K_{1}^{1 / 2} u_{t}(t)\right\}$ is relatively compact in $L^{2}(\Omega)$. To prove that $\bigcup_{t \geq 0}\{u(t)\}$ is relatively compact in $H^{1}(\Omega)$, we remark that

$$
\begin{aligned}
K_{1} u_{t}(t+h)-K_{1} u_{t}(t)-\int_{t}^{t+h} \Delta u(s) & d s+\int_{t}^{t+h} K_{2} u_{t}(s) d s \\
& =\int_{t}^{t+h}\left(g_{1}(s)-f(x, u(s))\right) d s .
\end{aligned}
$$


By using (F2), Lemma 12, $u_{t} \in L^{2}\left(\mathbf{R}^{+} ; L^{2}(\Omega)\right)$ and the fact that $\left(u, K_{1}^{1 / 2} u_{t}\right)$ is bounded with values in $H^{1}(\Omega) \times L^{2}(\Omega)$, we obtain

$$
\sup _{\substack{t \geq 0 \\ \delta \in[0,1]}}\left\|\int_{t}^{t+\delta} \Delta u(s) d s\right\|_{2}<\infty .
$$

By applying Lemma 13 (a) with $Y=H^{1}(\Omega)$ and $X=\{\phi \in$ $\left.H^{1}(\Omega) ; \Delta \phi \in L^{2}(\Omega)\right\}$, we obtain the claim.

5. Convergence of global weak solutions. In this section we study the long-time stabilization of global bounded solutions of (1), that is, we prove Theorems 7 and 8 . Let us recall that the $\omega$-limit set of a continuous function $u: \mathbf{R}^{+} \rightarrow H^{1}(\Omega)$ is defined by

$$
\begin{aligned}
& \omega(u)=\left\{\phi \in H^{1}(\Omega): \text { there exists } t_{n} \rightarrow+\infty\right. \\
& \text { such that } \left.\lim _{n \rightarrow \infty}\left\|u\left(t_{n}\right)-\phi\right\|_{H^{1}(\Omega)}=0\right\} .
\end{aligned}
$$

From well-known results on dynamical systems [12], if $u$ is a continuous function having in addition relatively compact range, then the $\omega$-limit set of $u$ is a non-empty, compact, and connected subset of $H^{1}(\Omega)$. Moreover, since our system has a continuous Lyapunov functional $G$, we prove the following lemma which is fundamental for the proof of Theorem 7 .

Lemma 14. Let $u$ be a global bounded weak solution of equation (1) and $v=(d / d t)\left(k *\left(u-u_{0}\right)\right)$. Then:

(i) The function $E$ is constant on $\omega(u)$, and

$$
E(\phi)=\lim _{t \rightarrow \infty} E(u(t))=E_{\infty}<\infty, \quad \text { for all } \phi \in \omega(u) .
$$

(ii) $\lim _{t \rightarrow \infty}\left\|K_{1}^{1 / 2} u_{t}\right\|_{2}=\lim _{t \rightarrow \infty} a *\|v\|_{\Gamma}^{2}=0$.

(iii) $E^{\prime}(\phi)=0$, for all $\phi \in \omega(u)$.

(iv) There exists a uniform Eojasiewicz exponent $\theta \in] 0,(1 / 2)], \beta>0$ and $T>0$ such that, for all $t \geq T$,

$$
\left|E(u(t))-E_{\infty}\right|^{1-\theta} \leq \beta\left\|E^{\prime}(u(t))\right\|_{*} .
$$


Proof. Let $\phi \in \omega(u)$. Then there exists an unbounded increasing sequence $\left(t_{n}\right)$ in $\mathbf{R}^{+}$such that $u\left(t_{n}\right) \rightarrow \phi$ in $H^{1}(\Omega)$. Since $u_{t} \in$ $L^{2}\left(\mathbf{R}^{+}, L^{2}(\Omega)\right)$, we have

$$
\begin{aligned}
u\left(t_{n}+s\right)=u\left(t_{n}\right)+\int_{t_{n}}^{t_{n}+s} u_{t}(\rho) d \rho \longrightarrow \phi & \\
& \text { in } L^{2}(\Omega) \text { for every } s \in[0,1] .
\end{aligned}
$$

This, together with the relative compactness of the trajectory in $H^{1}(\Omega)$, implies that $u\left(t_{n}+s\right) \rightarrow \phi$ in $H^{1}(\Omega)$ for every $s \in[0,1]$. Then, by continuity of $E, E\left(u\left(t_{n}+s\right)\right) \rightarrow E(\phi)$ in $H^{1}(\Omega)^{\prime}$ for every $s \in[0,1]$. Using the dominated convergence theorem,

$$
E(\phi)=\lim _{n \rightarrow \infty} \int_{0}^{1} E\left(u\left(t_{n}+s\right)\right) d s .
$$

Therefore, by integrating $G\left(t_{n}+\cdot\right)$ in $[0,1]$, we obtain

$$
E(\phi)=\lim _{n \rightarrow \infty} \int_{0}^{1} G\left(t_{n}+s\right) d s=G_{\infty},
$$

where we have used (T2), (G1), (52) and the following estimate:

$$
\begin{aligned}
\left|\int_{t_{n}}^{t_{n}+1}\left(g_{2}(s), a * v(s)\right)_{\Gamma} d s\right|^{2} \leq & \int_{t_{n}}^{t_{n}+1}\left\|g_{2}(s)\right\|_{\Gamma}^{2} d s \\
& +\|a\|_{L^{1}\left(\mathbf{R}^{+}\right)} \int_{t_{n}}^{t_{n}+1} a *\|v(s)\|_{\Gamma}^{2} d s .
\end{aligned}
$$

Since $\phi$ was chosen arbitrarily in $\omega(u)$, this implies that $E$ is constant on $\omega(u)$. Moreover, by the relative compactness of $u$ with values in $H^{1}(\Omega)$, we obtain $\lim _{t \rightarrow \infty} E(u(t))=G_{\infty}=E_{\infty}$. Then assertion (i) is proved. From this, the definition of $G$ and since $g_{2}(t)$ and the integral terms in $G$ tend to 0 as $t \rightarrow \infty$, we obtain assertion (ii).

In order to prove (iii), let $\phi \in \omega(u)$, and choose $t_{n} \rightarrow \infty$ such that $u\left(t_{n}\right) \rightarrow \phi$ in $H^{1}(\Omega)$. We have already seen that this implies $u\left(t_{n}+s\right) \rightarrow$ $\phi$ in $H^{1}(\Omega)$ for every $s \in[0,1]$. Hence, $E^{\prime}\left(u\left(t_{n}+s\right)\right) \rightarrow E^{\prime}(\phi)$ in $H^{1}(\Omega)^{\prime}$ for every $s \in[0,1]$. Finally, using the dominated convergence theorem, 
(T2), (T4), (ii) and (G1), we have, for all $\psi \in H^{1}(\Omega)$,

$$
\begin{aligned}
\left(E^{\prime}(\phi), \psi\right)_{H^{1}(\Omega)^{\prime}, H^{1}(\Omega)} & \int_{0}^{1}\left(E^{\prime}(\phi), \psi\right)_{H^{1}(\Omega)^{\prime}, H^{1}(\Omega)} d s \\
= & \lim _{n \rightarrow \infty} \int_{0}^{1}\left(E^{\prime}\left(u\left(t_{n}+s\right)\right), \psi\right)_{H^{1}(\Omega)^{\prime}, H^{1}(\Omega)} d s \\
= & \lim _{n \rightarrow \infty} \int_{0}^{1}\left(\int_{\Omega} \nabla u\left(t_{n}+s\right) \nabla \psi d x+\int_{\Omega} f\left(x, u\left(t_{n}+s\right)\right) \psi d x\right. \\
& \left.+\int_{\Gamma} \mu u\left(t_{n}+s\right) \psi d \sigma\right) d s \\
= & \lim _{n \rightarrow \infty} \int_{0}^{1}\left(-\frac{d}{d t} \int_{\Omega} K_{1} u_{t}\left(t_{n}+s\right) \psi d x\right. \\
& -\int_{\Omega}\left(K_{2} u_{t}-g_{1}\right)\left(t_{n}+s\right) \psi d x \\
& \left.-\int_{\Gamma}\left(v-g_{2}\right)\left(t_{n}+s\right) \psi d \sigma\right) d s \\
= & \lim _{n \rightarrow \infty}\left[\int _ { 0 } ^ { 1 } \left(\int_{\Omega}\left(-K_{2} u_{t}+g_{1}\right)\left(t_{n}+s\right) \psi d x\right.\right. \\
& \left.-\int_{\Gamma}\left(v-g_{2}\right)\left(t_{n}+s\right) \psi d \sigma\right) d s \\
= & 0 . \\
& \left.\int_{\Omega}\left(K_{1} u_{t}\left(t_{n}\right)-K_{1} u_{t}\left(t_{n}+1\right)\right) \psi d x\right] \\
& \\
& \\
& \\
&
\end{aligned}
$$

This proves (iii). Since $\omega(u)$ is compact, then there exists a finite family of open balls $B\left(\phi_{i}, \sigma_{i}\right)$ covering $\omega(u)$, where $\phi_{i} \in \omega(u)$ and $\sigma_{i}>0$ is such that $\left|E(\psi)-E_{\infty}\right| \leq 1$ for every $\psi \in B\left(\phi_{i}, \sigma_{i}\right)$. By Proposition 6 , and for all $i$, there exist constants $\theta_{i}$ and $\beta_{i}$ such that

$$
\left|E(\psi)-E_{\infty}\right|^{1-\theta_{i}} \leq \beta_{i}\left\|E^{\prime}(\psi)\right\|_{*},
$$

for every $\psi \in B\left(\phi_{i}, \sigma_{i}\right)$. Since $\lim _{t \rightarrow \infty} \operatorname{dist}(u(t), \omega(u))=0$, then there exists $T>0$ such that $u(t) \in \bigcup B\left(\phi_{i}, \sigma_{i}\right)$ for all $t \geq T$. Choosing $\beta=\sup \beta_{i}, \theta=\inf \theta_{i}$, we obtain (iv). 
After the previous preparation, we are ready to prove Theorem 7 .

Proof of Theorem 7 . Let $W_{0}(t): \mathbf{R}^{+} \rightarrow \mathbf{R}$ be the function defined by

$$
W_{0}(t)=G(t)-E_{\infty}+\varepsilon\left(E^{\prime}(u(t)), K_{1} u_{t}\right)_{*} \quad(t \geq 0) .
$$

Then, by (T3) and (T4), we have

$$
\begin{aligned}
\frac{d}{d t} W_{0}(t)= & \frac{d}{d t} G(t)+\varepsilon\left(E^{\prime \prime}(u) u_{t}, K_{1} u_{t}\right)_{*}+\varepsilon\left(E^{\prime}(u), K_{1} u_{t t}\right)_{*} \\
\leq & -\frac{k_{0}}{2}\left\|u_{t}\right\|_{2}^{2}-\frac{b_{\infty}}{2}\|v\|_{\Gamma}^{2}-\frac{\gamma}{4} a *\|v\|_{\Gamma}^{2} \\
& +\varepsilon\left(E^{\prime \prime}(u) u_{t}, K_{1} u_{t}\right)_{*}+\varepsilon\left(E^{\prime}(u),-E^{\prime}(u)-K_{2} u_{t}-v\right. \\
& \left.+g_{1}(t)+g_{2}(t)\right)_{*} .
\end{aligned}
$$

In order to estimate the term $\left(E^{\prime \prime}(u) u_{t}, K_{1} u_{t}\right)_{*}$ let $L: H^{1}(\Omega) \rightarrow H^{1}(\Omega)^{\prime}$ be the linear operator associated with the inner product on the space $H^{1}(\Omega)$ :

$$
(L u, v)_{H^{1}(\Omega)^{\prime}, H^{1}(\Omega)}=(u, v)_{H^{1}(\Omega)}=\int_{\Omega} \nabla u \nabla v d x+\int_{\Gamma} \mu u v d \sigma
$$

and let $K=L^{-1}$. We equip $H^{1}(\Omega)^{\prime}$ with the inner product:

$$
\left(g_{1}, g_{2}\right)_{*}=\left(K g_{1}, K g_{2}\right)_{H^{1}(\Omega)}, \quad g_{1}, g_{2} \in H^{1}(\Omega)^{\prime} .
$$

Note that $K \circ E^{\prime \prime}(v) \in \mathcal{L}\left(H^{1}(\Omega)\right)$. Moreover, by (63) and the definition of $E$, for all $u \in H^{1}(\Omega), v \in H^{1}(\Omega)$, we have

$$
K \circ E^{\prime \prime}(u) v=v+L^{-1}\left(\frac{\partial f}{\partial u}(x, u) v\right) .
$$

From this, the growth assumption on $f$ and the Sobolev embedding theorem, it is not difficult to deduce that the operator $K \circ E^{\prime \prime}(v)$ extends to a bounded linear operator on $L^{2}(\Omega)$ for every $v \in H^{1}(\Omega)$, and $K \circ E^{\prime \prime}: H^{1}(\Omega) \rightarrow \mathcal{L}\left(L^{2}(\Omega)\right)$ maps bounded sets into bounded sets.

In addition, for all $u \in L^{2}(\Omega), v \in H^{1}(\Omega)^{\prime}$, we have

$$
\begin{aligned}
(u, v)_{*} & =(K u, K v)_{H^{1}(\Omega)}=(L K u, K v)_{H^{1}(\Omega)^{\prime}, H^{1}(\Omega)} \\
& =(u, K v)_{H^{1}(\Omega)^{\prime}, H^{1}(\Omega)}=(u, K v)_{2} .
\end{aligned}
$$


Then:

$$
\begin{aligned}
\left(E^{\prime \prime}(u) u_{t}, K_{1} u_{t}\right)_{*} & =\left(K \circ E^{\prime \prime}(u) u_{t}, K_{1} u_{t}\right)_{2} \\
& \leq\left\|K \circ E^{\prime \prime}(u)\right\|_{\mathcal{L}\left(L^{2}(\Omega)\right)}\left\|K_{1}\right\|_{L^{\infty}(\Omega)}\left\|u_{t}\right\|_{2}^{2} \\
& \leq C\left\|u_{t}\right\|_{2}^{2} .
\end{aligned}
$$

Also, by the Cauchy-Schwarz inequality:

$$
\begin{aligned}
\left(E^{\prime}(u),-E^{\prime}(u)-K_{2} u_{t}-v+\right. & \left.g_{1}(t)+g_{2}(t)\right)_{*} \\
\leq & -\frac{1}{2}\left\|E^{\prime}(u)\right\|_{*}^{2}+C\left(\left\|u_{t}\right\|_{2}^{2}\right. \\
& \left.+\|v\|_{\Gamma}^{2}+\left\|g_{1}(t)\right\|_{2}^{2}+\left\|g_{2}(t)\right\|_{\Gamma}^{2}\right) .
\end{aligned}
$$

Combining (62)-(65) and choosing $\varepsilon>0$ small enough, we obtain

(66) $\frac{d}{d t} W(t) \leq-C\left(\left\|u_{t}\right\|_{2}^{2}+\left\|E^{\prime}(u)\right\|_{*}^{2}+\|v\|_{\Gamma}^{2}+a *\|v\|_{\Gamma}^{2}\right) \quad(t>0)$,

where $W: \mathbf{R}^{+} \rightarrow \mathbf{R}$ is the energy given by

$$
\begin{aligned}
W(t)= & \frac{1}{2}\left\|K_{1}^{1 / 2} u_{t}\right\|_{2}^{2}+E(u)-E_{\infty} \\
& +\frac{1}{2} a *\|v\|_{\Gamma}^{2}-\left(g_{2}, a * v\right)_{\Gamma}+\varepsilon\left(E^{\prime}(u(t)), K_{1} u_{t}\right)_{*} \\
& +\left(\frac{1}{2 k_{0}}-C_{\varepsilon}\right) \int_{t}^{\infty}\left\|g_{1}(s)\right\|_{2}^{2} d s \\
& +\left(d-C_{\varepsilon}\right) \int_{t}^{\infty}\left(\left\|g_{2}(s)\right\|_{\Gamma}^{2}+\left\|g_{2}^{\prime}(s)\right\|_{\Gamma}^{2}\right) d s,
\end{aligned}
$$

and $C_{\varepsilon}<\inf \left\{\left(1 / 2 k_{0}\right), d\right\}$. Thus, the function $W$ is nonincreasing and $\lim _{t \rightarrow \infty} W(t)=0$. It follows that $W(t) \geq 0$ for all $t \in \mathbf{R}^{+}$. If there exists a $T_{0} \geq 0$ such that $W\left(T_{0}\right)=0$, then $W(t)=0$ for all $t \geq T_{0}$. Therefore, by inequality $(66), u_{t}=0$ for all $t \geq T_{0}$, and the function $u$ is constant for $t \geq T_{0}$, that is, $u(t)=\phi$ for $t \geq T_{0}$. In this case, there remains nothing to prove. We may therefore suppose in the following that $W(t)$ is strictly positive on $\mathbf{R}^{+}$.

Now, Let $\theta$ be as in Lemma 14 (iv), and let $\theta_{0} \in(0, \theta]$ be such that

$$
(1+\delta)\left(1-\theta_{0}\right)>1
$$


that is, $\theta_{0}<\delta /(1+\delta)$. Note that $(61)$ is satisfied with $\theta$ replaced by $\theta_{0}$. Using Young's inequality, we deduce from the definition of $W$ and Lemma 9 that, for every $t \geq 0$,

$$
\begin{aligned}
W(t)^{1-\theta_{0}} \leq & C\left\{\left\|K_{1}^{1 / 2} u_{t}\right\|_{2}^{2\left(1-\theta_{0}\right)}+\left(a *\|v(t)\|_{\Gamma}^{2}\right)_{2}^{2\left(1-\theta_{0}\right) / 2}\right. \\
& +\left|E(u)-E_{\infty}\right|^{\left(1-\theta_{0}\right)}+\left\|g_{2}(t)\right\|_{\Gamma}+\left(a *\|v(t)\|^{2}\right)_{\Gamma}^{\left(1-\theta_{0}\right) /\left(2 \theta_{0}\right)} \\
& +\left(\int_{t}^{\infty}\left(\left\|g_{1}(s)\right\|_{2}^{2}+\left\|g_{2}(s)\right\|_{\Gamma}^{2}+\left\|g_{2}^{\prime}(s)\right\|_{\Gamma}^{2}\right) d s\right)^{1-\theta_{0}} \\
& \left.+\left\|K_{1} u_{t}\right\|_{2}^{\left(1-\theta_{0}\right) / \theta_{0}}+\left\|E^{\prime}(u)\right\|_{*}\right\}
\end{aligned}
$$

On the other hand, by assertions (ii) and (iv) from Lemma 14, there exists $T>0$ such that, for all $t \geq T$, we have

$$
\left\{\left\|K_{1}^{1 / 2} u_{t}\right\|_{2}+\left\|K_{1} u_{t}\right\|_{2}+\left(a *\|v(t)\|^{2}\right)_{2}^{1 / 2}\right\}<1
$$

and

$$
\left|E(u(t))-E_{\infty}\right|^{1-\theta_{0}} \leq \beta\left\|E^{\prime}(u(t))\right\|_{*} .
$$

Using this, $\left(\mathrm{G} 2^{\prime}\right)$ and the fact that $2\left(1-\theta_{0}\right) \geq 1$ and $\left(1-\theta_{0}\right) / \theta_{0} \geq 1$, we obtain, for all $t \geq T$,

$$
\begin{aligned}
W(t)^{1-\theta_{0}} \leq & C\left\{\left\|u_{t}\right\|_{2}+\left(a *\|v(t)\|_{\Gamma}^{2}\right)^{1 / 2}+\left\|E^{\prime}(u)\right\|_{*}\right. \\
& \left.+\left\|g_{2}(t)\right\|_{\Gamma}+(1+t)^{-(1+\delta)\left(1-\theta_{0}\right)}\right\} .
\end{aligned}
$$

Combining the last inequality and (66), we obtain

$$
-\frac{d}{d t} W(t)^{\theta_{0}}
$$

$$
=-\theta_{0} W(t)^{\theta_{0}-1} \frac{d}{d t} W(t)
$$

$$
\begin{aligned}
& \geq \frac{C\left(\left\|u_{t}\right\|_{2}^{2}+\left\|E^{\prime}(u)\right\|_{*}^{2}+\|v\|_{\Gamma}^{2}+a *\|v\|_{\Gamma}^{2}\right)}{\left\|u_{t}\right\|_{2}+\left(a *\|v(t)\|_{\Gamma}^{2}\right)^{1 / 2}+\left\|E^{\prime}(u)\right\|_{*}+\left\|g_{2}(t)\right\|_{\Gamma}+(1+t)^{-(1+\delta)\left(1-\theta_{0}\right)}} \\
& \geq C\left(\left\|u_{t}\right\|_{2}+\|v(t)\|_{\Gamma}+\left(a *\|v(t)\|_{\Gamma}^{2}\right)^{1 / 2}+\left\|E^{\prime}(u)\right\|_{*}\right) \\
& \quad-C\left(\left\|g_{2}(t)\right\|_{\Gamma}+(1+t)^{-(1+\delta)\left(1-\theta_{0}\right)}\right) .
\end{aligned}
$$


From this and the fact that the term $-(d / d t) W(t)^{\theta_{0}}+C\left(\left\|g_{2}(t)\right\|_{\Gamma}+\right.$ $\left.(1+t)^{-(1+\delta)\left(1-\theta_{0}\right)}\right)$ is integrable on $[T,+\infty)$, we obtain that $\left\|u_{t}\right\|_{2}$ is integrable on $[T,+\infty)$, which implies that $\lim _{t \rightarrow \infty} u(t, \cdot)$ exists in $L^{2}(\Omega)$. By the relative compactness of the range of $u$ in $H^{1}(\Omega)$, $\lim _{t \rightarrow \infty} u(t, \cdot)$ exists in $H^{1}(\Omega)$. This is the claim.

Proof of Theorem 8. The following lemma is used in the proof of the convergence rate to equilibrium. Its proof can be found in [4].

Lemma 15. Let $\zeta \in W_{\text {loc }}^{1,1}\left(\mathbf{R}^{+}, \mathbf{R}^{+}\right)$. We suppose that there exist constants $K_{1}>0, K_{2} \geq 0, k>1$ and $\lambda>0$ such that, for almost every $t \geq 0$, we have

$$
\zeta^{\prime}(t)+K_{1} \zeta(t)^{k} \leq K_{2}(1+t)^{-\lambda} .
$$

Then there exists a positive constant $m$ such that

$$
\zeta(t) \leq m(1+t)^{-\nu}, \quad \text { where } \nu=\inf \left\{\frac{1}{k-1}, \frac{\lambda}{k}\right\} .
$$

In order to prove Theorem 8, we proceed in two steps.

Step 1 (Polynomial decay). First, we note that the inequalities (69) and $(70)$ are satisfied when $\theta_{0}$ is replaced by the initial exponent $\theta$ given by Lemma 14 (iv). By using (69) together with Young's inequality, we obtain for every $t \in[T, \infty[$,

$$
\begin{aligned}
W(t)^{2(1-\theta)} \leq & C\left\{\left\|u_{t}\right\|_{2}^{2}+\left(a *\|v(t)\|_{\Gamma}^{2}\right)+\left\|E^{\prime}(u)\right\|_{*}^{2}\right. \\
& \left.+\left\|g_{2}(t)\right\|_{\Gamma}^{2}+(1+t)^{-2(1+\delta)(1-\theta)}\right\} .
\end{aligned}
$$

Using this, (G2) and (66), we obtain the following differential inequality for every $t \geq T$

$$
C \frac{d}{d t} W(t)+W(t)^{2(1-\theta)} \leq C(1+t)^{-2(1+\delta)(1-\theta)} .
$$

Then we may apply Lemma 15 in order to obtain

$$
W(t) \leq C(1+t)^{-\gamma},
$$


where $\gamma=\inf \{1 /(1-2 \theta), 1+\delta\}$. By again using (66), we have

$$
-\frac{d}{d t} W(t) \geq C\left\|u_{t}(t)\right\|_{2}^{2}
$$

Integrating this inequality over $[t, 2 t](t \geq T)$ and using (73), we obtain

$$
\int_{t}^{2 t}\left\|u_{t}(s)\right\|_{2}^{2} d s \leq C(1+t)^{-\gamma}
$$

Note that for every $t \in \mathbf{R}^{+}$,

$$
\int_{t}^{2 t}\left\|u_{t}(s)\right\|_{2} d s \leq t^{1 / 2}\left(\int_{t}^{2 t}\left\|u_{t}(s)\right\|_{2}^{2} d s\right)^{1 / 2} .
$$

It follows that

$$
\int_{t}^{2 t}\left\|u_{t}(s)\right\|_{2} d s \leq C(1+t)^{(1-\gamma) / 2} \quad \text { for every } t \geq T .
$$

Therefore, we obtain for every $t \geq T$,

$$
\begin{aligned}
\int_{t}^{\infty}\left\|u_{t}(s)\right\|_{2} d s & \leq \sum_{k=0}^{\infty} \int_{2^{k} t}^{2^{k+1} t}\left\|u_{t}(s)\right\|_{2} d s \\
& \leq C \sum_{k=0}^{\infty}\left(2^{k} t\right)^{(1-\gamma) / 2} \leq C(1+t)^{(1-\gamma) / 2} .
\end{aligned}
$$

Then, for all $t \geq T$,

$$
\begin{gathered}
\|u(t)-\phi\|_{2} \leq \int_{t}^{\infty}\left\|u_{t}(s)\right\|_{2} d s \leq C(1+t)^{-\xi}, \\
\text { where } \xi=\inf \left\{\frac{\theta}{1-2 \theta}, \frac{\delta}{2}\right\} .
\end{gathered}
$$

Step 2 (Exponential decay). Suppose that $g_{1}=0$ and $g_{2}=0$. Then (72) becomes

$$
-\frac{d}{d t} W(t) \geq C W(t)^{2(1-\theta)}
$$


Since $W(t)>0$, for sufficiently large times $t$, we obtain from this inequality that

$$
\begin{cases}(-1 /(1-2 \theta))(d / d t) W(t)^{-(1-2 \theta)} \leq-C & \text { if } \theta \in(0,(1 / 2)) \\ (d / d t)(\ln W(t)) \leq-C & \text { if } \theta=1 / 2\end{cases}
$$

Hence, integrating these differential inequalities, we obtain that there exists a constant $C>0$ such that, for every large $t>0$,

$$
\begin{cases}W(t) \leq C(1+t)^{-1 /(1-2 \theta)} & \text { if } \theta \in(0,(1 / 2)), \\ W(t) \leq C e^{-C t} & \text { if } \theta=1 / 2 .\end{cases}
$$

Note that the inequality (70) (when $g_{1}=g_{2}=0$ ) implies, for every $s \geq T$,

$$
-\frac{d}{d t} W(t)^{\theta} \geq C\left\|u_{t}(t)\right\|_{2}
$$

Integrating this inequality on the interval $[t, \infty)(t \geq T)$, we obtain

$$
\|u(t)-\phi\|_{2} \leq \int_{t}^{\infty}\left\|u_{t}(s)\right\|_{2} d s \leq C W(t)^{\theta} .
$$

This inequality together with inequality (74) implies the claim.

Acknowledgments. The author would like to thank Professor Ralph Chill for his many valuable suggestions and the referees for their important remarks and comments.

\section{REFERENCES}

1. S. Aizicovici and E. Feireisl, Long-time stabilization of solutions to a phasefield model with memory, J. Evol. Equat. 1 (2001), 69-84.

2. S. Aizicovici and H. Petzeltová, Asymptotic behaviour of solutions of a conserved phase-field system with memory, J. Int. Equat. Appl. 15 (2003), 217-240.

3. F. Alabau-Boussouira, J. Prüss and R. Zacher, Exponential and polynomial stability of a wave equation for boundary memory damping with singular kernels, C.R. Acad. Sci. Paris 347 (2009), 277-282.

4. I. Ben Hassen, Decay estimates to equilibrium for some asymptotically autonomous semilinear evolution equations, Asymp. Anal. 69 (2010), 31-44.

5. I. Ben Hassen and L. Chergui, Convergence of global and bounded solutions of some non-autonomous second order evolution equations with nonlinear dissipation, J. Dyn. Diff. Equat. 23 (2011), 315-332.

6. M.M. Cavalcanti, V.N. Domingos Cavalcanti, J.S. Prates Filho and J.A. Soriano, Existence and uniform decay rates for viscoelastic problems with nonlinear boundary damping, J. Diff. Int. Equat. 14 (2001), 85-116. 
7. M.M. Cavalcanti, V.N. Domingos Cavalcanti and M.L. Santos, Existence and uniform decay rates of solutions to a degenerate system with memory conditions at the boundary, Appl. Math. Comp. 150 (2004), 439-465.

8. R. Chill and E. Fašangová, Convergence to steady states of solutions of semilinear evolutionary integral equations, Calc. Var. P.D.E. 22 (2005), 321-342.

9. R. Chill and M.A. Jendoubi, Convergence to steady states in asymptotically autonomous semilinear evolution equation, Nonl. Anal. 53 (2003), 1017-1039.

10. C. Dafermos, Asymptotic stability in viscoelasticity, Arch. Rat. Mech. Anal. 37 (1970), 297-308.

11. E. Feireisl and F. Simondon, Convergence for semilinear degenerate parabolic equations in several space dimensions, J. Dyn. Diff. Equat. 12 (2000), 647-673.

12. A. Haraux, Systèmes dynamiques dissipatifs et applications, Masson, Paris, 1990.

13. - Almost periodic forcing for a wave equation with a nonlinear, local damping term, Proc. Roy. Soc. Edinb. 94 (1983), 195-212.

14. A. Haraux and M.A. Jendoubi, Convergence of bounded weak solutions of the wave equation with dissipation and analytic nonlinearity, Calc. Var. P.D.E. 9 (1999), 95-124.

15. S.Z. Huang and P. Takác, Convergence in gradient-like systems which are asymptotically autonomous and analytic, Nonl. Anal. 46 (2001), 675-698.

16. M.A. Jendoubi, Convergence of global and bounded solutions of the wave equation with linear dissipation and analytic nonlinearity, J. Diff. Equat. 144 (1998), 302-312.

17. - A simple unified approach to some convergence theorem of L. Simon, J. Funct. Anal. 153 (1998), 187-202.

18. M.L. Santos, Asymptotic behavior of solutions to wave equations with a memory conditions at the boundary, Elect. J. Diff. Equat. 78 (2001), 1-11.

19. L. Simon, Asymptotics for a class of non-linear evolution equation with applications to geometric problem, Ann. Math. 118 (1983), 525-571.

20. V. Vergara and R. Zacher, Lyapunov functions and convergence to steady state for differential equations of fractional order. Math. Z. 259 (2008), 287-309.

21. H. Yassine, Asymptotic behaviour and decay rate estimates for a class of semilinear evolution equations of mixed order, Nonl. Anal. 74 (2011), 2309-2326.

22. — Existence and asymptotic behavior of solutions to semilinear wave equations with nonlinear damping and dynamical boundary condition, J. Dyn. Diff. Equat. (2012), doi 10.1007/s10884-012-9258-1.

23. R. Zacher, Convergence to equilibrium for second order differential equations with weak damping of memory type, Adv. Diff. Equat. 14 (2009), 749-770.

Université de Lorraine, Laboratoire de Mathématiques et Applications de Metz et CNRS, UMR 7122, Bât. A, Ile du Saulcy, 57045 Metz Cedex 1, France

Email address: yassine@univ-metz.fr 